\title{
NUMERICAL INTEGRATION FOR HIGH ORDER PYRAMIDAL FINITE ELEMENTS ${ }^{*, * *}$
}

\author{
Nilima Nigam ${ }^{1}$ AND Joel Phillips ${ }^{2}$
}

\begin{abstract}
We examine the effect of numerical integration on the accuracy of high order conforming pyramidal finite element methods. Non-smooth shape functions are indispensable to the construction of pyramidal elements, and this means the conventional treatment of numerical integration, which requires that the finite element approximation space is piecewise polynomial, cannot be applied. We develop an analysis that allows the finite element approximation space to include non-smooth functions and show that, despite this complication, conventional rules of thumb can still be used to select appropriate quadrature methods on pyramids. Along the way, we present a new family of high order pyramidal finite elements for each of the spaces of the de Rham complex.
\end{abstract}

Mathematics Subject Classification. 65N30,65D30.

Received November 23, 2010. Revised June 16, 2011.

Published online October 12, 2011

\section{INTRODUCTION}

Our aim in this paper is to present a careful account of the variational crime arising from the use of numerical quadrature with arbitrarily high order pyramidal finite elements.

Pyramidal finite elements are used in applications as "glue" in heterogeneous meshes containing hexahedra, tetrahedra and prisms. In prior work, [17], we presented a family of high-order finite element spaces that approximate each of the spaces of the de Rham complex, on a pyramidal element. Various constructions of high order pyramidal elements have been proposed [7,11,12,17,21,22]. A useful summary of the approaches taken for $H^{1}$-conforming elements is given by Bergot et al. [3], who also provide some motivating numerical results for the performance of methods based on meshes containing pyramidal elements. If they are to be used to implement stable mixed methods, such elements should also satisfy a commuting diagram property. In addition to our work, an approach based on the theory of local exact sequences, was suggested by Zaglmayr, [21], and are summarised in [9].

In [17] we demonstrated that approximation spaces consisting only of polynomials would not suffice. For example, there is no polynomial whose trace is the lowest order bubble on one triangular face and zero on all

\footnotetext{
Keywords and phrases. Finite elements, quadrature, pyramid.

* The work of NN was supported by the Natural Sciences and Engineering Research Council of Canada, and the Canada Research Chairs program.

** JP was supported by a Canada Natural Sciences and Engineering Research Council of Canada graduate scholarship and by Engineering and Physical Sciences Research Council grant EP/H004009/1.

1 Department of Mathematics, Simon Fraser University, 8888 University Drive, Burnaby, BC V5A 1S6, Canada.

nigam@math.sfu.ca

2 Department of Mathematics, University College London, Main Campus Gower Street Bloombury, WC1E 6BT, London, UK. joel.phillips@ucl.ac.uk
} 
other faces of a pyramid. The need to include non-polynomial functions in our finite element approximation spaces arose because we must match polynomial traces on all faces with data from neighbouring tetrahedra or hexahedra. We used rational functions on the pyramid in our construction, as have other authors [17,19,20].

In practice, numerical quadrature will be necessary for matrix assembly. Recent work on the use of quadrature schemes for finite element methods has been focussed on issues of efficiency and fast implementation, see e.g. [14]. The classical analysis of the effect of quadrature, see, e.g. [5,6], has the lesser objective, nicely summed up in [6], of

"[giving] sufficient conditions on the quadrature scheme which insure that the effect of the numerical integration does not decrease [the] order of convergence".

Typically the stiffness matrix and load vector in the finite element formulation of a given PDE are computed using an inexact numerical quadrature. The errors introduced by these quadrature rules can be treated as a variational crime and, provided the finite element approximation spaces are piece-wise polynomials, are wellunderstood. The approximation spaces we presented in [17] were shown to include complete sets of polynomials and so, at first glance, one might expect the classical analysis of errors due to numerical quadrature should hold in the case of the pyramidal finite elements as well [3]. Somewhat surprisingly, this is not the case. As we will see in Example 2.4, the importance of the rational functions in constructing interpolants means that it is not possible to achieve global estimates of the consistency error by summing element-wise estimates that only deal with polynomials.

Our exclusive focus in this paper, therefore, is a careful analysis of the errors introduced by quadrature when pyramidal finite elements are used. The main challenge in this analysis arises from the fact that pyramidal elements necessarily include functions other than polynomials and that the rational functions favoured by us and most other authors are not arbitrarily smooth. This means that the Bramble-Hilbert Lemma cannot be applied directly.

In this paper we address this particular difficulty. We demonstrate that an $n$th order quadrature rule can be used for the integration of bilinear forms involving $n$th order elements without decreasing the overall order of covergence of the method. Specifically, we introduce a new definition of a family of high order pyramidal finite elements and show that what Stroud calls the "conical product formulae" [18] are an appropriate choice of quadrature rule. We present a unified analysis of the consistency error due to using these quadrature rules for each of the approximation spaces of the discrete de Rham complex.

We have chosen to restrict our analysis to meshes composed solely of parallelogram-based pyramids. We will refer to a general such pyramid as an affine pyramid. Note that the restriction to purely pyramidal meshes is for the sake of exposition; in practice, the necessity and utility of pyramidal elements is evident in meshes comprised of tetrahedral, prismatic and parallelipiped elements as well. Our accounting of quadrature errors is based on local estimates and the elements on the pyramid are conforming so extending our arguments to mixed meshes is straightforward. On the other hand, the extension to meshes that include general quadrilateral-based pyramids, for which there is no affine map from a single reference pyramidal element, may not be straightforward and we make no claims about the applicability of our analysis to this case.

In Section 2 we summarise the classical theory for the effect of numerical integration on finite element methods and show that it is insufficient when, as in the case of pyramidal elements, a basis contains functions that are not arbitrarily smooth. As a first step towards overcoming this, we derive a generalisation of the standard Bramble-Hilbert argument.

Section 3 introduces a framework that will allow us to unify our analysis for discrete approximations to each of the spaces of the de Rham complex. We think of members of these spaces as differential forms, but we will introduce a notation that should be accessible to readers who prefer to think in terms of vector calculus. We also introduce our reference pyramid(s).

In Section 4 we introduce a new family of approximation spaces that can be used to construct pyramidal finite elements for each space of the de Rham complex and in Section 5 we demonstrate that the conical product rules are a good choice to use with these (and our original) pyramidal finite elements. 
Proofs of the properties of the new family of pyramidal elements are contained in an appendix. In most cases these are either similar to, or based on, those for our original family of elements.

In what follows, we shall use the notation $P^{k}$ to denote polynomials of maximal total degree $k$.

\section{BACKGROUND}

\subsection{Quadrature errors on pyramidal finite elements cannot be analyzed using the classical theory}

Let $K$ be a pyramid in $\mathbb{R}^{3}$ with triangular faces and a parallelogram base. In [17] we constructed pyramidal finite elements for each of the spaces in the de Rham complex, $H^{1}(K), H(\operatorname{curl}, K), H(\operatorname{div}, K)$ and $L^{2}(K)$, that also satisfy a commuting diagram property. Members of these four spaces can be regarded as $s$-forms where $s \in\{0,1,2,3\}$ (forms are quickly introduced in Sect. 3). Accordingly, in this paper we will denote the $k$ th order approximation spaces on a pyramid $K$, as $\mathcal{U}_{k}^{(s)}(K)$. We recall the definitions of these spaces in the appendix, but for this section, we only need the fact that these spaces included rational functions whose high-order derivatives may have poles on the boundary of the pyramid $K$. We will demonstrate that this causes the classical theory of the effect of quadrature on finite elements to break down, and derive a generalisation of the Bramble Hilbert Lemma that will help us to resolve the problem.

Let us first recall the standard framework for analyzing the variational crime due to quadrature. Let $V$ be a Hilbert space, $a: V \times V \rightarrow \mathbb{R}$ be an elliptic bilinear form and let the problem of finding $u \in V$ such that

$$
a(u, v)=f(v) \quad \forall v \in V
$$

be well-posed for all functionals $f \in V^{\prime}$. A discrete version of this problem is to find $u_{h} \in V_{h}$ such that

$$
a_{h}\left(u_{h}, v\right)=f(v) \quad \forall v \in V_{h},
$$

where $V_{h}$ is an approximating subspace of $V$ and $a_{h}$ approximates $a$ using numerical integration. ${ }^{1}$ When $V_{h}$ is assembled using polynomials of degree $k$ on shape-regular elements with maximum diameter, $h$, the analysis of the effect of the numerical integration is well-known, and may be found in $[5,6]$. An analysis for mixed problems can be found in [10]. The conclusion is the same: in order to preserve an $O\left(h^{k}\right)$ approximation error, each (numerically computed) bilinear form must satisfy an $O\left(h^{k}\right)$ consistency error estimate.

For an example, take an elliptic bilinear form $a: H_{0}^{1}(\Omega) \times H_{0}^{1}(\Omega) \rightarrow \mathbb{R}$, defined as

$$
a(u, v)=\int_{\Omega} A(\nabla u, \nabla v) \mathrm{d} x
$$

where $A$ a uniformly positive definite covariant tensor with entries in $W^{k, \infty}(\Omega)$.

Assume that $V_{h} \subset H_{0}^{1}(\Omega)$ is some approximation space assembled using $k$ th order polynomial finite elements. Let $S_{h, k, \Omega}(\cdot)$ be a quadrature rule, which satisfies $S_{h, k, \Omega}\left(\partial_{i} u \partial_{j} v\right)=\int_{\Omega}\left(\partial_{i} u \partial_{j} v\right)$ for any $i$ and $j$ and all pairs of functions $u, v \in V_{h}$. Let $a_{h}(u, v)=S_{h, k, \Omega}(A(\nabla u, \nabla v))$. It is shown in [6], page 179, that the solution of (2.2) will satisfy the error estimate:

$$
\left\|u-u_{h}\right\|_{1} \leq C h^{k}\left(|u|_{k+1}+\|A\|_{k, \infty}\|u\|_{k+1}\right) .
$$

This result is contingent on an estimate of the consistency error:

$$
\sup _{w_{h} \in V_{h}} \frac{\left|a\left(\Pi_{h} u, w_{h}\right)-a_{h}\left(\Pi_{h} u, w_{h}\right)\right|}{\left\|w_{h}\right\|_{1}} \leq C h^{k}\|A\|_{k, \infty}\|u\|_{k+1},
$$

\footnotetext{
${ }^{1}$ We choose not to consider the effect of approximating $f(\cdot)$ by some $f_{h}(\cdot)$ using numerical integration because it is no different on the pyramid than for other elements. Error estimates may be obtained by applying the standard argument and using Theorem 5.2 .
} 
where $\Pi_{h}: H_{0}^{1}(\Omega) \rightarrow V_{h}$ is a bounded interpolation operator. The constant $C=C(\Omega, k)$ is independent of $h$. The key ingredient in the proof of the consistency error estimate, (2.4) is a local estimate:

Theorem 2.1 (see [6], Thm. 4.1.4). Given some $k \geq 0$ and a simplex, $K \in \mathcal{T}_{h}$, where $\mathcal{T}_{h}$ is a shape-regular mesh with maximum element diameter $h$, assume that there exists a quadrature rule on $K$ which is exact for $P^{2 k-2}$. That is, the error functional $E_{k, K}$ for the quadrature rule satisfies $E_{k, K}(\psi)=0$ for any polynomial $\psi \in P^{2 k-2}(K)$. Then there exists a constant $C$ independent of $K$ and $h$ such that

$$
\begin{aligned}
& \forall A \in W^{k, \infty}(K), \quad \forall p, q \in P^{k}(K) \\
& \left.\mid E_{k, K}\left(A \partial_{i} p \partial_{j} q\right)\right) \mid \leq C h^{k}\|A\|_{k, \infty, K}\left\|\partial_{i} p\right\|_{k-1, K} \cdot\left\|\partial_{j} q\right\|_{0, K} .
\end{aligned}
$$

This theorem, in turn, is proved by combining a scaling argument with the following famous result from [4].

Theorem 2.2 (Bramble-Hilbert Lemma). Let $\Omega \subset \mathbb{R}^{n}$ be open with Lipschitz-continuous boundary. For some integer $k \geq 0$ and $p \in[0, \infty]$ let the linear functional, $f: W^{k+1, p}(\Omega) \rightarrow \mathbb{R}$ have the property that $\forall \psi \in P^{k}(\Omega)$, $f(\psi)=0$. Then there exists a constant $C(\Omega)$ such that

$$
\forall v \in W^{k+1, p}(\Omega), \quad|f(v)| \leq C(\Omega)\|f\|_{W^{k+1, p}(\Omega)^{\prime}}|v|_{k+1, p, \Omega}
$$

where $\|\cdot\|_{W^{k+1, p}(\Omega)}$ is the operator norm.

We observe that the partial derivatives that appear in Theorem 2.1 are in fact components of the gradient and since $\nabla \mathcal{U}_{k}^{(0)}(K) \subset \mathcal{U}_{k}^{(1)}(K)$ we might conjecture the following analogous statement:

Conjecture 2.3. Let $K \in \mathcal{T}_{h}$ be a pyramid. Let $A \in W^{k, \infty}(K)$. Suppose that there exists a quadrature rule (with error functional $E_{k, K}$ ) that integrates products of shape functions in $\mathcal{U}_{k}^{(1)}(K)$ exactly. Then

$$
\begin{aligned}
& \forall v, w \in \mathcal{U}_{k}^{(1)}(K) \\
& \left|E_{k, K}(A v w)\right| \leq C h^{k}\|A\|_{k, \infty, K}\|v\|_{k-1, K}\|w\|_{0, K}
\end{aligned}
$$

This conjecture (and the analogs for $\mathcal{U}_{k}^{(s)}(K), s=0,2,3$ which are used for the analysis of mixed problems) is in fact true, but useless. We are indeed able to construct a quadrature rule which is exact for products of shape functions. The problem is that we cannot differentiate basis functions in $\mathcal{U}_{k}^{(0)}(K)$ arbitrarily, unlike the situation for purely polynomial spaces. The following example is illustrative:

Example 2.4. Let $\hat{K}$ denote the reference pyramid:

$$
\hat{K}=\{(\xi, \eta, \zeta) \mid 0 \leq \zeta \leq 1,0 \leq \xi, \eta \leq \zeta\}
$$

Take the $\mathcal{U}_{k}^{(0)}(\hat{K})$ shape function associated with the base vertex, $(1,1,0)$ :

$$
v(\xi, \eta, \zeta)=\frac{\xi \eta}{1-\zeta}
$$

The third partial $\zeta$-derivative $\frac{\partial^{3} v}{\partial \zeta^{3}} \notin L^{2}(\hat{K})$ :

$$
\int_{\hat{K}}\left(\frac{\partial^{3} v}{\partial \zeta^{3}}\right)^{2} \mathrm{~d} \hat{x}=\int_{0}^{1} \int_{0}^{1-\zeta} \int_{0}^{1-\zeta}\left(\frac{-6 \xi \eta}{(1-\zeta)^{4}}\right)^{2} \mathrm{~d} \xi \mathrm{d} \eta \mathrm{d} \zeta=\int_{0}^{1} \frac{9}{(1-\zeta)^{2}} \mathrm{~d} \zeta
$$

Hence $v \notin H^{3}(\hat{K})$. 
Similar issues arise for each of the finite element spaces $\mathcal{U}_{k}^{(s)}(\hat{K})$. This means that a direct application of the argument in [6], Section 4.1, would fail when we attempt to use the Bramble-Hilbert Lemma (Thm. 2.2) to obtain the estimate

$$
\left|\Pi_{k, \hat{K}}^{(s)} u\right|_{r, \hat{K}} \leq C|u|_{r, \hat{K}} \quad \forall r \in\{0, \ldots, k\}
$$

where $\Pi_{k, \hat{K}}^{(s)}$ is any bounded interpolant to $\mathcal{U}_{k}^{(s)}(\hat{K})$.

An attempt is made to avoid this problem in [3] by using the additional projector $\pi_{r}: H^{r+1}(K) \rightarrow P^{r}$ satisfying

$$
\forall p \in P^{r}(K) \quad \pi_{r} p=p
$$

on each element, $K$. This allows element-wise estimates to be established. Unfortunately, there is no conforming interpolant onto element-wise polynomials for pyramidal elements (see [17] or [20]). In particular, there will be discontinuities at the element boundaries, which means that $\left\|u-\pi_{r} u\right\|_{1, \Omega}$ cannot be bounded. The alternative interpretation of $\pi_{r}$ as a global projection onto polynomials would not allow the element-wise estimates to be obtained.

\subsection{Interpolation errors revisited}

Fortunately, not all of the members of each $\mathcal{U}_{k}^{(s)}(K)$ behave as badly as the function $v$ defined in (2.7). There are subspaces of polynomials and of rational functions that are more regular. For example, we readily observe that, again taking the function $v$ defined in $(2.7), v(\xi, \eta, \zeta) \xi^{r} \in H^{r+2}(\hat{K}) \cap \mathcal{U}_{r}^{(0)}(\hat{K})$. In order to analyze the consistency error introduced via quadrature, we will thus need an analog of the interpolation error estimate (2.9) that allows us to use this regularity when it is available.

Theorem 2.5. Let $K \subset \mathbb{R}^{n}$ be an open set with Lipschitz boundary. Fix $\alpha \geq 0$ and let $k \geq \alpha$ be an integer. Suppose that:

- $R^{k} \subset H^{\alpha}(K)$ is a finite dimensional space which includes all polynomials of degree $k$;

- $\Pi: H^{\alpha}(K) \rightarrow R^{k}$ is a bounded linear projection;

- There exist $V_{r} \subset H^{r}(K)$ for each $r \in\{0, \ldots, k\}$ such that we can decompose

$$
R^{k}=V_{0} \oplus \cdots \oplus V_{k}
$$

That is, given $u \in H^{k}(K)$, its interpolant, $\Pi u \in R^{k}$, may be decomposed into unique functions, $v_{r} \in V_{r}$,

$$
\Pi u=v_{0}+\cdots+v_{k}
$$

Then we have the following estimates for some of the functions, $v_{r}$ :

- For each $r$ satisfying $\alpha \leq r \leq k$ :

$$
\left|v_{r}\right|_{r} \leq C|u|_{r}
$$

- If, additionally, $\tilde{P}^{r} \subset V_{r}$, where the space $\tilde{P}^{r}$ consists of polynomials of homogeneous degree, $r$, then for each $r$ satisfying $\alpha \leq r+1 \leq k$ :

$$
\left|v_{r}\right|_{r} \leq C|u|_{r+1}+|u|_{r}
$$


Proof. For a given $r \geq \alpha$, write $W_{r}=V_{r}+P^{r-1}$. The space $R^{k}$ contains all polynomials of degree $k$, so $W_{r} \subset R^{k}$ and we can write $W_{r}=V_{r} \oplus\left(P^{r-1} \cap \bigoplus_{i \neq r} V_{i}\right)$. This means that we can find a surjective linear projection, $\Psi_{r}: R^{k} \rightarrow W_{r}$ that satisfies $\left(\Psi_{r} \circ \Pi\right) u=v_{r}+p$ for some $p \in P^{r-1}$.

$\Psi_{r}$ is a linear map between finite spaces, so the operator $\left(I-\Psi_{r} \circ \Pi\right): H^{r}(K) \rightarrow W_{r} \subset H^{r}(K)$ is bounded. Both $\Psi_{r}$ and $\Pi$ are projections, so $\operatorname{ker}\left(I-\Psi_{r} \circ \Pi\right)=W_{r} \supset P^{r-1}$. Therefore we can use the Bramble-Hilbert Lemma to see that

$$
\left\|\left(I-\Psi_{r} \circ \Pi\right) u\right\|_{r} \leq C|u|_{r},
$$

so $\left|u-v_{r}-p\right|_{r} \leq C|u|_{r}$, which in turn implies that

$$
\left|v_{r}\right|_{r} \leq C|u|_{r}+|u|_{r}+|p|_{r}=(C+1)|u|_{r}
$$

The proof of (2.12) follows a similar argument. The operator $\left(I-\Psi_{r} \circ \Pi\right): H^{r+1}(K) \rightarrow W_{r} \subset H^{r}(K)$ is bounded because $r+1 \leq k$. The additional condition, $\tilde{P}^{r} \subset V_{r}$, means that $P^{r} \subset W_{r}$ and so $P^{r} \subset \operatorname{ker}\left(I-\Psi_{r} \circ \Pi\right)$.

\section{Definitions}

\subsection{Differential forms, reference coordinate systems}

From now on, we will want to make general statements that apply to approximations to each of the spaces of the de Rham complex. It is natural and increasingly popular to use differential forms and the exterior calculus in such a discussion $[1,2]$, and since we work with pyramids in $\mathbb{R}^{3}$, calculations are simplified. We refer the reader to [1], Section 2.3, for a comprehensive account of the exterior calculus on $\mathbb{R}^{n}$.

Let $\Omega$ be an open set in $\mathbb{R}^{3}$. A point, $x \in \Omega$, has coordinates $\left(x^{i}\right)_{i=0 \ldots 3}$ in terms of some global coordinate functions. We can define $\Lambda^{(s)}(\Omega)$, the space of differential $s$-forms on $\Omega$, as consisting of elements of the form

$$
u=\sum_{1 \leq \alpha_{1}<.<\alpha_{s} \leq 3} u_{\alpha} \mathrm{d} x^{\alpha_{1}} \wedge \cdots \wedge \mathrm{d} x^{\alpha_{s}}
$$

where each $u_{\alpha} \in C^{\infty}(\Omega)$ and the multi-index, $\alpha=\alpha_{1} \cdots \alpha_{s}$. If we allow $u_{\alpha} \in H^{r}(\Omega), u$ is a member of the Hilbert space $H^{r} \Lambda^{(s)}(\Omega)$ with norm and semi-norm

$$
\|u\|_{r, \Omega}^{2}:=\sum_{\alpha}\left\|u_{\alpha}\right\|_{H^{r}(\Omega)}^{2}, \quad|u|_{r, \Omega}^{2}:=\sum_{\alpha}\left|u_{\alpha}\right|_{H^{r}(\Omega)}^{2} .
$$

If $r=0$, we denote $H^{0} \Lambda^{(s)}(\Omega) \equiv L^{2} \Lambda^{(s)}(\Omega)$.

It is conventional to think of differential forms in terms of proxy fields. The spaces $\Lambda^{(0)}(\Omega)$ and $\Lambda^{(3)}(\Omega)$ are isomorphic to the scalar field, $C^{\infty}(\Omega)$. The spaces $\Lambda^{(1)}(\Omega)$ and $\Lambda^{(2)}(\Omega)$ are isomorphic to the vector field, $\left(C^{\infty}(\Omega)\right)^{3}$. With these identifications, the exterior derivatives, $d: \Lambda^{(s)}(\Omega) \rightarrow \Lambda^{(s+1)}(\Omega)$ for $s=0,1,2$ become the familiar grad, curl and div, and the de Rham complex

$$
0 \longrightarrow \Lambda^{(0)}(\Omega) \stackrel{d}{\longrightarrow} \Lambda^{(1)}(\Omega) \stackrel{d}{\longrightarrow} \Lambda^{(2)}(\Omega) \stackrel{d}{\longrightarrow} \Lambda^{(3)}(\Omega) \longrightarrow 0
$$

becomes

$$
0 \longrightarrow C^{\infty}(\Omega) \stackrel{\text { grad }}{\longrightarrow}\left(C^{\infty}(\Omega)\right)^{3} \stackrel{\text { curl }}{\longrightarrow}\left(C^{\infty}(\Omega)\right)^{3} \stackrel{\text { div }}{\longrightarrow} C^{\infty}(\Omega) \longrightarrow 0
$$


Using the exterior derivatives, we have an elegant correspondence between the familiar $H^{1}(\Omega), H(\operatorname{curl}, \Omega), H$ $(\operatorname{div}, \Omega)$ and $L^{2}(\Omega)$ spaces for the proxy-fields and the spaces $\mathcal{H}^{s}(\Omega), s=0,1,2,3$ respectively, with

$$
\mathcal{H}^{(s)}(\Omega):=\left\{u \in L^{2} \Lambda^{(s)}(\Omega) \mid \mathrm{d} u \in L^{2} \Lambda^{(s+1)}(\Omega)\right\}, \quad\|u\|_{\mathcal{H}^{(s)}}^{2}:=\|u\|_{L^{2} \Lambda^{(s)}}^{2}+\|\mathrm{d} u\|_{L^{2} \Lambda^{(s+1)}}^{2} .
$$

Define $\Theta^{(s)}(\Omega)$ to be the space of all (covariant) tensors, $A: \Lambda^{(s)}(\Omega) \times \Lambda^{(s)}(\Omega) \rightarrow C^{\infty}(\Omega)$ that can be defined in terms of the pointwise representation,

$$
A(u, v)(x):=A^{\alpha \beta}(x) u_{\alpha}(x) v_{\beta}(x) \quad \forall u, v \in \Lambda^{(s)}(\Omega), x \in \Omega
$$

where we are using the Einstein summation convention, $A^{\alpha \beta} u_{\alpha} v_{\beta}:=\sum_{\alpha, \beta \in \Upsilon_{s}} A^{\alpha \beta} u_{\alpha} v_{\beta}$. We will insist that $A^{\alpha \beta}$ is anti-symmetric in the first $s$ and second $s$ components, which makes the representation unique. For $A \in \Theta^{(s)}(\bar{\Omega})$, we define

$$
|A|_{k, \infty, \Omega}^{2}:=\sum_{1 \leq i, j \in\left(\begin{array}{l}
3 \\
s
\end{array}\right)}\left|A^{i j}\right|_{W^{k, \infty}(\Omega)}^{2}, \quad \quad\|A\|_{k, \infty, \Omega}^{2}:=\sum_{r=0}^{k}|A|_{r, \infty, \Omega}^{2}
$$

and define $W^{r, \infty} \Theta^{(s)}(\Omega)$ to be the completion of $\Theta^{(s)}(\bar{\Omega})$ in $\|\cdot\|_{r, \infty, \Omega}$.

We will decorate the subscripts (and superscripts) of proxies with symbols to indicate the coordinate system that is being used to determine the components of the proxy fields. Given some $u \in \Lambda^{(s)}(\Omega)$, $u_{i^{\prime}}$ is the $i$ th component of its proxy in the coordinate system $x^{\prime}=\left(x^{1^{\prime}}, x^{2^{\prime}}, x^{3^{\prime}}\right)$. We will also write $u^{\prime}=\left(u_{i^{\prime}}\right)_{i \in\left\{1, \ldots\left(\begin{array}{l}3 \\ s\end{array}\right)\right\}}$ to indicate all the components of the vector (or scalar) field. Differential forms are contravariant, so the components transform as:

$$
u_{\alpha^{\prime}}=\sum_{\alpha \in \Upsilon_{s}} \frac{\partial x^{\alpha_{1}}}{\partial x^{\alpha_{1}^{\prime}}} \cdots \frac{\partial x^{\alpha_{s}}}{\partial x^{\alpha_{s}^{\prime}}} u_{\alpha}
$$

The components of a covariant tensor, $A \in \Theta^{(s)}(\Omega)$ transform as:

$$
A^{\alpha^{\prime} \beta^{\prime}}=\sum_{\alpha, \beta \in \Upsilon_{s}} \frac{\partial x^{\alpha_{1}^{\prime}}}{\partial x^{\alpha_{1}}} \cdots \frac{\partial x^{\alpha_{s}^{\prime}}}{\partial x^{\alpha_{s}}} \frac{\partial x^{\beta_{1}^{\prime}}}{\partial x^{\beta_{1}}} \cdots \frac{\partial x^{\beta_{s}^{\prime}}}{\partial x^{\beta_{s}}} A^{\alpha \beta}
$$

For a coordinate change, $x=\phi\left(x^{\prime}\right)$, the weights appearing in the contravariant and covariant transformation rules, (3.3) and (3.4), can be written in terms of the entries of a $\left(\begin{array}{l}3 \\ s\end{array}\right) \times\left(\begin{array}{l}3 \\ s\end{array}\right)$ matrix, $w_{\phi}^{(s)}$. We choose to let $w_{\phi}^{(s)}$ to be the weight in the covariant transformation so that, for $u \in \Lambda^{(s)}(\Omega)$

$$
\sum_{i \leq i^{\prime} \leq\left(\begin{array}{l}
3 \\
s
\end{array}\right)}\left(w_{\phi}^{(s)}\right)_{i, i^{\prime}} u_{i^{\prime}}=u_{i} \quad \forall 1 \leq i \leq\left(\begin{array}{l}
3 \\
s
\end{array}\right) .
$$

The weights can be calculated in terms of the Jacobian, $D \phi$ :

$$
w_{\phi}^{(0)}=1, \quad w_{\phi}^{(1)}=D \phi^{-1^{t}}, \quad w_{\phi}^{(2)}=\operatorname{det}\left(D \phi^{-1}\right) D \phi, \quad w_{\phi}^{(3)}=\operatorname{det}\left(D \phi^{-1}\right) .
$$

It is useful to identify a specific and convenient coordinate system. The use of a reference coordinate system is a familiar concept. In the engineering literature, shape functions for finite elements on simplices are often defined in terms of barycentric coordinates. After thinking of a scalar or vector field as a proxy to a differential form, 
the use of the reference coordinate system to study a shape function is analogous to mapping the differential form to a reference element using a pullback.

Let $\mathcal{T}$ be a partition of $\Omega$ where every $K \in \mathcal{T}$ is the image of a simple reference domain, $\hat{K} \subset \mathbb{R}^{n}$, under a diffeomorphism $\phi_{K}: \hat{K} \rightarrow K$. On each $K$, the reference coordinates, $\hat{x}=\left(x^{\hat{i}}\right)_{\hat{i}=1 \ldots n}$ of any point $x \in K$, are given by $\hat{x}=\phi_{K}^{-1}(x)$. Given $u \in \Lambda^{(s)}(K)$ and $A \in \Theta^{(s)}(K)$ the reference coordinate system induces new sets of components $u_{\hat{\alpha}}$ and $A^{\hat{\alpha} \hat{\beta}}$, using the rules (3.3) and (3.4). If $A \in \Theta^{(s)}(\Omega)$ and $u, v \in \Lambda^{(s)}(\Omega)$, we note that $A^{\alpha \beta}(x) u_{\alpha}(x) v_{\beta}(x)=A^{\hat{\alpha} \hat{\beta}}(\hat{x}) u_{\hat{\alpha}}(\hat{x}) v_{\hat{\beta}}(\hat{x})$ is just a 0 -form. Hence we have the important change of variables formula on each element, $K$ :

$$
\int_{K} A^{\alpha \beta} u_{\alpha} v_{\beta} \mathrm{d} \mathbf{x}=\int_{\hat{K}} A^{\hat{\alpha} \hat{\beta}} u_{\hat{\alpha}} v_{\hat{\beta}} \operatorname{det}\left(D \phi_{K}\right) \mathrm{d} \hat{x}
$$

where $D \phi_{K}$ is the Jacobian of $\phi_{K}$ and $\operatorname{det}\left(D \phi_{K}\right)$ is the determinant of the Jacobian.

The exterior derivative is an intrinsic property of any manifold. This means that it is independent of coordinates; equivalently, the exterior derivative commutes with coordinate transformation.

\subsection{Reference pyramidal element and scaling laws}

To contain the proliferation of indices, we will use the notation $(\xi, \eta, \zeta)$ for the reference coordinates $\left(x^{\hat{1}}, x^{\hat{2}}, x^{\hat{3}}\right)$. The reference domain is defined as the pyramid:

$$
\hat{K}=\{(\xi, \eta, \zeta) \mid 0 \leq \zeta \leq 1,0 \leq \xi, \eta \leq \zeta\}
$$

Suppose each $\phi_{K}$ satisfies

$$
\left\|D \phi_{K}\right\| \leq h \quad \text { and } \quad\left\|D \phi_{K}^{-1}\right\| \leq \frac{\rho}{h}
$$

for some $h>0$ and $\rho \geq 1$. For any $u \in H^{k} \Lambda^{(s)}(K)$ and $A \in W^{k, \infty} \Theta^{(s)}(K)$, we have the inequalities

$$
\begin{aligned}
& \frac{1}{C \rho^{k+s}} \frac{h^{k+s}}{\operatorname{det}\left(D \phi_{K}\right)^{1 / 2}}|u|_{k, K} \leq|u|_{k, \hat{K}} \leq C \frac{h^{k+s}}{\operatorname{det}\left(D \phi_{K}\right)^{1 / 2}}|u|_{k, K} \\
& \frac{1}{C \rho^{k}} h^{k-2 s}|A|_{k, \infty, K} \leq|A|_{k, \infty, \hat{K}} \leq C \rho^{2 s} h^{k-2 s}|A|_{k, \infty, K}
\end{aligned}
$$

for some constant $C=C(k, n)$ which is independent of $h$. These can be deduced from the standard scaling argument for Sobolev semi-norms of functions (see, for example, [6]) combined with the transformation rules, (3.3) and (3.4) and the observation that (3.8) implies that $\frac{\partial x^{\alpha_{i}}}{\partial \hat{x}^{\alpha_{j}}} \leq h$ and $\frac{\partial \hat{x}^{\hat{\alpha}_{j}}}{\partial x^{\alpha_{i}}} \leq \frac{\rho}{h}$ for all $i, j$.

As in [17] we will also use the infinite pyramid,

$$
K_{\infty}=\{(x, y, z) \mid 0 \leq x, y \leq 1,0 \leq z \leq \infty\}
$$

as a tool to help analyse and define the pyramidal elements. The finite and infinite pyramids may be identified using the projective mapping $\phi: K_{\infty} \rightarrow \hat{K}$ defined as

$$
\phi:(x, y, z) \mapsto\left(\frac{x}{1+z}, \frac{y}{1+z}, \frac{z}{1+z}\right),
$$

which can also be thought of as a change of coordinates and so induces the infinite pyramid coordinate system defined as $\tilde{x}=\phi^{-1} \hat{x}$. We shall usually write $\tilde{x}=(x, y, z)$. 
The corresponding weights in the change of coordinates transformation rule 3.5 can be calculated explicitly:

$$
\begin{aligned}
& w_{\phi}^{(0)}=1 \\
& w_{\phi}^{(1)}=D \phi^{-1^{t}}=(1+z)\left(\begin{array}{ccc}
1 & 0 & 0 \\
0 & 1 & 0 \\
x & y & 1+z
\end{array}\right) \\
& w_{\phi}^{(2)}=\operatorname{det}\left(D \phi^{-1}\right) D \phi=(1+z)^{2}\left(\begin{array}{ccc}
1+z & 0 & -x \\
0 & 1+z & -y \\
0 & 0 & 1
\end{array}\right) \\
& w_{\phi}^{(3)}=\operatorname{det}\left(D \phi^{-1}\right)=(1+z)^{4} .
\end{aligned}
$$

Suppose that $\left(\mathcal{T}_{h}\right)_{h>0}$ is a family of shape-regular partitions of $\Omega$, where every $K \in \mathcal{T}_{h}$ is affine equivalent to $\hat{K}$. For a given element $K \in \mathcal{T}_{h}, u \in \Lambda^{(s)}(\bar{K})$ and $A \in \Theta^{(s)}(\bar{K})$, we define the reference semi-norms ${ }^{2}$ :

$$
|u|_{k, \hat{K}}:=\sum_{1 \leq \hat{i} \leq\left(\begin{array}{l}
3 \\
s
\end{array}\right)}\left|u_{\hat{i}}\right|_{H^{k}(\hat{K})}, \quad|A|_{k, \infty, \hat{K}}:=\sum_{1 \leq \hat{i}, \hat{j} \leq\left(\begin{array}{l}
3 \\
s
\end{array}\right)}\left|A^{\hat{i} \hat{j}}\right|_{W^{k, \infty}(\hat{K})} .
$$

\section{A FAMILY OF PYRAMIDAL FINITE ELEMENTS}

Theorem 2.5 allows us to contemplate an argument based on the Bramble Hilbert Lemma for approximation spaces containing non-smooth basis functions. The spaces $\mathcal{U}_{k}^{(s)}(K)$ can, in fact, be decomposed in the manner necessary for Theorem 2.5 to apply but, unfortunately, we are not able to use it to analyse their consistency error directly ${ }^{3}$. To overcome this problem we will identify subspaces $\mathcal{R}_{k}^{(s)}(K)$ of $\mathcal{U}_{k}^{(s)}(K)$ that allow us to construct finite elements which are also conforming, and which satisfy a commuting diagram property. We will show the new spaces contain the same complete space of polynomials and which are compatible with neighbouring tetrahedral and hexahedral elements (See [3] for an analysis of the $H^{1}$-conforming case). They thus have the same approximability properties as $\mathcal{U}_{k}^{(s)}(K)$.

The easiest way to establish the properties of these new elements is to utilize the properties of the original $\mathcal{U}_{k}^{(s)}(K)$ elements established in [17]. Since these proofs are interesting, but distracting from our main argument, we have included them in an appendix.

\subsection{Construction}

We start the construction of the new approximation spaces in the infinite pyramid coordinate system introduced in Section 3.2 using spaces of $k$-weighted polynomials, $Q_{k}^{[l, m]}$, which we define in terms of basis functions $\frac{x^{a} y^{b}}{(1+z)^{c}}, a, b$ and $c$ are non-negative integers.

$$
Q_{k}^{[l, m]}=\operatorname{span}\left\{\frac{x^{a} y^{b}}{(1+z)^{c}}: \quad c \leq k, a \leq c+l-k, b \leq c+m-k\right\}
$$

\footnotetext{
${ }^{2}$ These are the norms induced by the metric in which the reference coordinates are orthonormal. They are used in the scaling argument in Section 5 .

${ }^{3}$ If we applied the argument in Section 5 directly to the spaces, $\mathcal{U}_{k}^{(s)}(K)$, it would fail at equation (5.8) in the proof of Lemma 5.3.
} 
These spaces can be characterised via a decomposition into spaces of exactly $r$-weighted tensor product polynomials,

$$
Q_{k}^{[l, m]}=\bigoplus_{r=0}^{k} Q_{r}^{r+l-k, r+m-k, 0}
$$

where $Q_{r}^{l, m, n}[x, y, z]$ is spanned by the $\operatorname{set}^{4}\left\{\frac{x^{a} y^{b} z^{c}}{(1+z)^{r}}, 0 \leq a \leq l, 0 \leq b \leq m, 0 \leq c \leq n\right\}$.

It is also helpful to observe that $\frac{x^{a} y^{b}}{(1+z)^{c}} \mapsto \xi^{a} \eta^{b}(1-\zeta)^{c-a-b}$ under the coordinate transformation, $(\eta, \xi, \zeta)=$ $\phi(x, y, z)$ given by (3.11). So if the representation in the infinite pyramid coordinate system of some polynomial $f(\hat{x})$ is $\tilde{f} \in Q_{k}^{[l, m]}$ then $f$ is at most degree $k$ in $(\xi, \eta, \zeta)$ and at most degree $l$ and $m$ in $(\xi, \zeta)$ and $(\eta, \zeta)$ respectively.

Definition 4.1. We define the spaces $\mathcal{R}_{k}^{(s)}$ on $K_{\infty}$, for $s=\{0,1,2,3\}$ and $k \geq 0$ as

$$
\begin{aligned}
& \mathcal{R}_{k}^{(0)}=Q_{k}^{[k, k]} \\
& \mathcal{R}_{k}^{(1)}=\left(Q_{k+1}^{[k-1, k]} \times Q_{k+1}^{[k, k-1]} \times\{0\}\right) \oplus\left\{\nabla u: u \in Q_{k}^{[k, k]}\right\}, \\
& \mathcal{R}_{k}^{(2)}=\left(\{0\} \times\{0\} \times Q_{k+2}^{[k-1, k-1]}\right) \oplus\left\{\nabla \times u: u \in\left(Q_{k+1}^{[k-1, k]} \times Q_{k+1}^{[k, k-1]} \times\{0\}\right)\right\} \\
& \mathcal{R}_{k}^{(3)}=Q_{k+3}^{[k-1, k-1]}
\end{aligned}
$$

The decomposition in the definitions (4.3) means that with the identification made as in Section 3.1, the exterior derivatives, $d: \mathcal{R}_{k}^{(s)} \rightarrow \mathcal{R}_{k}^{(s+1)}$ are well defined. The gradient is injective on $Q^{[k, k]} / \mathbb{R} ;$ the curl is injective on $\left(Q_{k+1}^{[k-1, k]} \times Q_{k+1}^{[k, k-1]} \times\{0\}\right)$ and the divergence is a bijection from $\left(\{0\} \times\{0\} \times Q_{k+2}^{[k-1, k-1]}\right)$ to $Q_{k+3}^{[k-1, k-1]}$, so the sequence,

$$
\mathbb{R} \longrightarrow \mathcal{R}_{k}^{(0)} \stackrel{\nabla}{\longrightarrow} \mathcal{R}_{k}^{(1)} \stackrel{\nabla \times}{\longrightarrow} \mathcal{R}_{k}^{(2)} \stackrel{\nabla \cdot}{\longrightarrow} \mathcal{R}_{k}^{(3)} \longrightarrow 0
$$

is exact. We can readily define these approximation spaces on any $K \in \mathcal{T}_{h}$.

Definition 4.2. For a given $s \in\{0,1,2,3\}$ and $k \geq 0$, we define the approximation space $\mathcal{R}_{k}^{(s)}(K)$ on a pyramid, $K$, as those differential forms whose infinite pyramid coordinate representation lie in $\mathcal{R}_{k}^{(s)}$ :

$$
\mathcal{R}_{k}^{(s)}(K)=\left\{u \in \Lambda^{(s)}(K):\left(u_{\tilde{i}}\right) \in \mathcal{R}_{k}^{(s)}\right\}
$$

Let us first show that despite the presence of rational functions, these approximation spaces conform on each element:

Lemma 4.3. For a given $K$ and $s \in\{0,1,2,3\}$ let $u \in \mathcal{R}_{k}^{(s)}(K)$. Each component $u_{\hat{i}}$ (where $\left.\hat{i} \in\left\{1, \ldots,\left(\begin{array}{l}3 \\ s\end{array}\right)\right\}\right)$ of $u$ in the reference coordinate system satisfies

$$
u_{\hat{i}} \circ \phi \in Q_{k}^{k, k, k}
$$

This means that

$$
\mathcal{R}_{k}^{(s)}(K) \subset \mathcal{H}^{(s)}(K)
$$

\footnotetext{
${ }^{4}$ If $l, m$ or $n$ is negative then $Q^{l, m, n}:=\{0\}$.
} 
Proof. The relationship between the representations of $u$ in the reference and infinite pyramid coordinate systems is given by equation (3.5): $\hat{u} \circ \phi=w_{\phi}^{(s)} \tilde{u}$, where the weights, $w_{\phi}^{(s)}$, are given by (3.12). To establish (4.6), each $s \in\{0,1,2,3\}$ needs to be dealt with as a separate case.

When $s=0$, the weight, $w_{\phi}^{(0)}=1$ and it is clear from (4.3a) that $\mathcal{R}_{k}^{(0)} \subset Q_{k}^{k, k, k}$. When $s=1$, inspection of (4.3b) reveals that $\mathcal{R}_{k}^{(1)} \subset Q_{k+1}^{k-1, k, k} \times Q_{k+1}^{k, k-1, k} \times Q_{k+1}^{k, k, k-1}$. The weight, $w_{\phi}^{(1)}=(1+z)\left(\begin{array}{ccc}1 & 0 & 0 \\ 0 & 1 & 0 \\ x & y & 1+z\end{array}\right)$, so $w_{\phi}^{(1)} \tilde{u} \in Q_{k}^{k, k, k} \times Q_{k}^{k, k, k} \times Q_{k}^{k, k, k}$. The cases $s=2$ and $s=3$ follow similarly.

Since $Q_{k}^{k, k, k} \subset L^{\infty}\left(K_{\infty}\right)$ each $u_{\hat{i}} \circ \phi$ is bounded on $K_{\infty}$, which means that $u_{\hat{i}}$ is bounded on $\hat{K}$ and therefore $u_{i}$ is bounded on $K$. Hence $\|u\|_{0, K}$ is finite. By $(4.4), d u \in \mathcal{R}_{k}^{(s+1)}(K)$, so $\|d u\|_{0, K}$ is finite too and $u \in \mathcal{H}^{(s)}(K)$.

The approximation spaces may be equipped with interpolation operators. We summarize the properties of the resulting pyramidal elements, and defer proofs to the appendix:

(1) Approximability: each space $\mathcal{R}_{k}^{(s)}(K)$ contains the same complete polynomial subspace that was shown to be present in the space $\mathcal{U}_{k}^{(s)}(K)$ in [17]. This is established in Lemma 4.4

(2) Compatibility: the elements are compatible with existing high order tetrahedral and hexahedral elements (as first outlined in [16] for $s=1,2$ ). Lemma 4.5 establishes this.

(3) Commutativity: equipped with interpolation operators $\Phi_{k, K}^{(s)}$ defined via degrees of freedom, the elements satisfy a commuting diagram property, Theorem 4.7 .

Lemma 4.4. If $K$ is an affine (i.e. parallelogram-based) pyramid then, for $k \geq 1$,

$$
\begin{aligned}
& P^{k} \subset \mathcal{R}_{k}^{(0)}(K) \\
& \left(P^{k-1}\right)^{\left(\begin{array}{l}
3 \\
s
\end{array}\right)} \subset \mathcal{R}_{k}^{(s)}(K) \quad s \in\{1,2,3\} .
\end{aligned}
$$

Lemma 4.5. Let $K$ be a pyramid. For each $s \in\{0,1,2\}$ there is a trace operator that takes elements of $\mathcal{H}^{(s)}(K)$ to some distribution on the boundary, $\partial K$. The image of $\mathcal{R}_{k}^{(s)}(K)$ under this operator consists of all traces of elements of $\mathcal{H}^{(s)}(K)$ whose restriction to each triangular or quadrilateral face of $K$ is the traces of a function belonging to the approximation space of the corresponding kth order Lagrange, Nedelec edge or Nedelec face element.

Lemma 4.6. The new approximation spaces are subspaces of the original approximation spaces.

$$
\mathcal{R}_{k}^{(s)} \subseteq \mathcal{U}_{k}^{(s)} . \quad s \in\{0,1,2,3\}
$$

In [17], we had defined degrees of freedom for $\mathcal{U}_{k}^{(s)}(K)$ which ensured unisolvency; these induced interpolants $\Pi_{k, K}^{(s)}: H^{2} \Lambda^{(s)}(K) \rightarrow \mathcal{U}_{k}^{(s)}(K)$. In part consequence of Lemmas 4.4 and 4.6, we can define bounded linear interpolants $\Xi_{k, K}^{(s)}: \mathcal{U}_{k}^{(s)}(K) \rightarrow \mathcal{R}_{k}^{(s)}(K)$ which leave the data on the boundary of $K$ invariant. The composition of these two interpolants, $\Phi_{k, K}^{(s)}:=\Xi_{k, K}^{(s)} \circ \Pi_{k, K}^{(s)}$ provides interpolants which yield the commuting diagram property. The definition and properties of $\Xi_{k, K}^{(s)}$ are technical, and are postponed until the appendix, where we also prove the following Theorem which establishes the commuting diagram property:

Theorem 4.7. There exist bounded interpolation operators, $\Phi_{k, K}^{(s)}: H^{2} \Lambda^{(s)}(K) \rightarrow \mathcal{R}_{k}^{(s)}(K)$ that satisfy the identity $d \circ \Phi_{k, K}^{(s)}=\Phi_{k, K}^{(s)} \circ d$. 
As a consequence, for a given $k$, we can assemble a global approximation space,

$$
\mathcal{S}_{h}^{(s)}=\left\{v \in \mathcal{H}^{s}(\Omega): v \mid K \in \mathcal{R}_{k}^{(s)}(K) \forall K \in \mathcal{T}_{h}\right\}
$$

and define a global bounded interpolation operator $\Phi_{h}^{(s)}: H^{2} \Lambda^{(s)}(\Omega) \rightarrow \mathcal{S}_{h}^{(s)}$ by $\left.\left(\Phi_{h}^{(s)} u\right)\right|_{K}:=\Phi_{k, K}^{(s)}\left(\left.u\right|_{K}\right)$ for all $K \in \mathcal{T}_{h}$.

\subsection{Decomposition}

The next step is to show that the spaces $\mathcal{R}_{k}^{(s)}(K)$ possess a decomposition that is compatible with Theorem 2.5.

Definition 4.8. Let $K \in \mathcal{T}_{h}$ be a pyramid, and $s \in\{0,1,2,3\}$. For each $r \geq 0$ we define

$$
\mathcal{X}_{r, k}^{(s)}(K)=\left\{v \in \mathcal{R}_{k}^{(s)}(K): v_{\hat{i}} \circ \phi \in Q_{r}^{r+1, r+1,0}\right\}
$$

That is, $\mathcal{X}_{r, k}^{(s)}(K)$ is the subspace consisting of $s$-forms in $\mathcal{R}_{k}^{(s)}(K)$ whose components are exactly $r$-weighted when composed with $\phi: K_{\infty} \rightarrow \hat{K}$.

Note that although the domain of $v_{\hat{i}} \circ \phi$ is $K_{\infty}$, the condition is on the components in the reference coordinate system, $v_{\hat{i}}$, rather than the infinite pyramid coordinate system $v_{\tilde{i}}$. In effect, what we are saying is that each $\mathcal{X}_{r, k}^{(s)}(K)$ is spanned by $s$-forms whose components have the form

$$
e(\xi, \eta, \zeta)=\xi^{a} \eta^{b}(1-\zeta)^{r-a-b}, a, b \leq r+1
$$

Lemma 4.9. For an affine pyramid, $K$ and for each $s \in\{0,1,2,3\}$ and $k \geq 1$, each of the spaces $\mathcal{X}_{r, k}^{(s)}(K)$ satisfies the criterion for $V_{r},(2.10)$, from Theorem 2.5. In fact,

$$
\mathcal{X}_{r, k}^{(s)}(K) \subset H^{r+1} \Lambda^{(s)}(K)
$$

Additionally, the Sobolev semi-norm $|\cdot|_{r, K}$ is actually a norm on each space $\mathcal{X}_{r, k}^{(s)}(K)$.

Proof. Let $u \in \mathcal{X}_{r, k}^{(s)}(K)$. Each $u_{\hat{i}}$ can be written in terms of functions, $e(\xi, \eta, \zeta)=\xi^{a} \eta^{b}(1-\zeta)^{r-a-b}$. When $a+b>r$, these will be rational functions with a singularity at $\zeta=1$. We need to understand their differentiability on the finite pyramid. Let $\gamma=\left(\gamma_{1}, \gamma_{2}, \gamma_{3}\right)$ be a multi-index. The partial derivative,

$$
\frac{\partial^{\gamma} e}{\partial \hat{x}^{\gamma}}=C \xi^{a-\gamma_{1}} \eta^{b-\gamma_{2}}(1-\zeta)^{r-b-a-\gamma_{3}}
$$

where $C=C(\gamma, a, b, r)$ is a (possibly zero) constant dependent only on $\gamma, a, b$ and $r$. Hence

$$
\begin{aligned}
\int_{\hat{K}}\left(\frac{\partial^{\gamma} e}{\partial \hat{x}^{\gamma}}\right)^{2} & =C \int_{0}^{1} \int_{0}^{1-\zeta} \int_{0}^{1-\zeta} \xi^{2 a-2 \gamma_{1}} \eta^{2 b-2 \gamma_{2}}(1-\zeta)^{2 r-2 b-2 a-2 \gamma_{3}} \mathrm{~d} \xi \mathrm{d} \eta \mathrm{d} \zeta \\
& =C \int_{0}^{1}(1-\zeta)^{2\left(r+1-\gamma_{1}-\gamma_{2}-\gamma_{3}\right)} \mathrm{d} \zeta
\end{aligned}
$$

This integral is finite if $r+1-|\gamma|>-1 / 2$, so $e \in H^{\lfloor r+3 / 2-\epsilon\rfloor}(\hat{K})$. By affine equivalence of $K$ and $\hat{K}$, $u_{i} \in H^{\lfloor r+3 / 2-\epsilon\rfloor}(K) \subset H^{r+1}(K)$, and therefore $u \in H^{r+1} \Lambda^{(s)}(K)$.

Finally, (4.9) shows that each $e(\xi, \eta, \zeta)$ is either a rational function, or a polynomial of degree exactly $r$, so $|e|_{r, \hat{K}} \neq 0$. Hence $u \neq 0$ implies that $|u|_{r, K} \neq 0$ and so $|\cdot|_{r, K}$ is a norm on $\mathcal{X}_{r, k}^{(s)}(K)$. 
Theorem 4.10. For an affine pyramid, $K$ and for each $s \in\{0,1,2,3\}$ and $k \geq 1$, each of the spaces $\mathcal{R}_{k}^{(s)}(K)$ may be decomposed:

$$
\mathcal{R}_{k}^{(s)}(K)=\mathcal{X}_{0, k}^{(s)}(K) \oplus \cdots \oplus \mathcal{X}_{k, k}^{(s)}(K)
$$

Proof. The decomposition (4.2) and the definitions in (4.6) make the claim look plausible. The details are technical and are left to the appendix.

\section{Numerical INTEGRATION ON PYRAMIDAL ELEMENTS}

In this section we will prove the main consistency result of this paper, a version of Theorem 2.1 for bilinear forms applied to each of the families of pyramidal elements. We start by presenting some pyramidal quadrature formulae and showing that they integrate products of pyramidal shape functions exactly.

\subsection{Conical product rule}

Quadrature rules on the pyramid can be deduced as special cases of the conical product rule presented by Stroud $[13,18]$. Let $\hat{K}$ be the reference pyramid, and $f \in C(\hat{K})$. We are specifically interested in the case when $f$ is a product of pair of $k t h$-degree polynomials, $k \geq 0$. Following his work, we define the quadrature scheme of degree $2 k+1$ to approximate $\int_{\hat{K}} f \mathrm{~d} x$ as follows:

$$
S_{k, \hat{K}}(f):=\sum_{i, j, l} f\left(\xi_{i}\left(1-\zeta_{l}\right), \xi_{j}\left(1-\zeta_{l}\right), \zeta_{l}\right) \lambda_{i} \lambda_{j} \mu_{l} .
$$

Stroud showed that given $k \geq 0$, a sufficient condition for $S(k, \hat{K})(p)=\int_{\hat{K}} p \mathrm{~d} \hat{x}$ for any polynomial, $p \in$ $P^{2 k+1}(\hat{x})$, is that the two quadrature schemes given by the points $\xi_{i}$ and $\zeta_{l}$ with respective weights $\lambda_{i}$ and $\mu_{l}$ satisfy

$$
\begin{aligned}
& \sum_{i} \lambda_{i} g\left(\xi_{i}\right)=\int_{0}^{1} g(x) \mathrm{d} x \quad \forall g \in P^{2 k+1}, \\
& \sum_{i} \mu_{i} h\left(\zeta_{i}\right)=\int_{0}^{1}(1-z)^{2} h(z) \mathrm{d} z \quad \forall h \in P^{2 k+1} .
\end{aligned}
$$

The $k+1$ point Gauss-Legendre quadrature rule can be used to generate nodes $\xi_{i}$ and weights $\lambda_{i}$ that make (5.2a) exact for polynomials of degree $2 k+1$. The $k+1$ point Gauss-Jacobi scheme based on the shifted Jacobi polynomial $^{5}, P_{k+1}^{(2,0)}$, generates nodes $\zeta_{i}$ and weights $\mu_{i}$ that make $(5.2 \mathrm{~b})$ exact for polynomials of degree $2 k+1$. This is the quadrature rule we shall use for the pyramidal elements. With this, the quadrature error,

$$
\begin{aligned}
E_{k, \hat{K}}(f) & :=S_{k, \hat{K}}(f)-\int_{\hat{K}} f(\hat{x}) \mathrm{d} \hat{x} \\
& =0 \quad \text { if } f \in P^{2 k+1} .
\end{aligned}
$$

\footnotetext{
${ }^{5}$ The Jacobi polynomials, $P_{n}^{(a, b)}(s), n \geq 0$, are typically defined on the interval $[-1,1]$. Under the change of variables, $s=2 t-1$, we obtain the shifted Jacobi polynomials which are orthogonal with respect to the weight $(1-t)^{a} t^{b}$ on the interval $[0,1]$.
} 
When $f \in C(K)$, where $K$ is a pyramid equipped with a change of coordinates $\phi_{K}: \hat{K} \rightarrow K$, we can define the quadrature and error functionals:

$$
\begin{aligned}
& S_{k, K}(f):=S_{k, \hat{K}}\left(\left|D \phi_{K}\right| \hat{f}\right) \sim \int_{K} f(x) \mathrm{d} x, \\
& E_{k, K}(f):=E_{k, \hat{K}}\left(\left|D \phi_{K}\right| \hat{f}\right)=S_{k, K}(f)-\int_{K} f(x) \mathrm{d} x,
\end{aligned}
$$

where $\hat{f}=f \circ \phi_{K}$, i.e. the expression of $f$ in the reference coordinate system, $\hat{x}$.

\subsection{Quadrature errors}

Lets look at the effect of the conical product rules on our approximation spaces. Following (5.3), the rule $S_{k, K}(\cdot)$ is exact for functions in $P^{2 k+1}$ on the pyramid, $K$. Hence, if $u$ and $v$ are polynomials of degree $k$ then their product, $u v \in P^{2 k}$ so, clearly, $S_{k, \hat{K}}(u v)=\int_{\hat{K}} u v$.

We first demonstrate which functions our quadrature scheme integrates exactly.

Lemma 5.1. Suppose that $f$ be defined on the pyramid $K$, and that the representation of $f$ in the infinite pyramid coordinate system, $\tilde{f}=f \circ \phi_{K} \circ \phi$, lies in the space $Q_{2 k+1}^{2 k+1,2 k+1,2 k+1}$. Then the quadrature scheme, $S_{k, K}$ is exact for $f$ :

$$
S_{k, K}(f)=\int_{K} f \mathrm{~d} x
$$

Proof. It suffices to consider functions $p$ with a representation in the infinite pyramid coordinate system:

$$
\tilde{p}(x, y, z)=\frac{x^{a} y^{b}}{(1+z)^{c}} \quad 0 \leq a, b, c \leq 2 k+1,
$$

since these monomials span the space $Q_{2 k+1}^{2 k+1,2 k+1,2 k+1}$. In finite reference coordinates, $p$ has the form $\hat{p}(\xi, \eta, \zeta)=$ $\xi^{a} \eta^{b}(1-\zeta)^{c-a-b}$, and so, using (5.1):

$$
\begin{aligned}
S_{k, K}(p) & =S_{k, \hat{K}}\left(\operatorname{det}\left(D \phi_{K}\right) \hat{p}\right) \\
& =\operatorname{det}\left(D \phi_{K}\right) \sum_{i, j, l} \xi_{i}^{a}\left(1-\zeta_{l}\right)^{a} \xi_{j}^{b}\left(1-\zeta_{l}\right)^{b}\left(1-\zeta_{l}\right)^{c-a-b} \lambda_{i} \lambda_{j} \mu_{l} \\
& =\operatorname{det}\left(D \phi_{K}\right) \sum_{i} \lambda_{i} \xi_{i}^{a} \sum_{j} \lambda_{j} \xi_{i}^{b} \sum_{l} \mu_{l}\left(1-\zeta_{l}\right)^{c} \\
& =\operatorname{det}\left(D \phi_{K}\right) \int_{0}^{1} s^{a} \mathrm{~d} s \int_{0}^{1} t^{b} \mathrm{~d} t \int_{0}^{1}(1-\zeta)^{c+2} \mathrm{~d} \zeta .
\end{aligned}
$$

The last step is justified because each of the sums is a quadrature rule applied to a polynomial of degree $\leq 2 k+1$ and so we can apply (5.2a) and (5.2b). We use the change of variables $\xi=(1-\zeta) s$ and $\eta=(1-\zeta) t$ to obtain:

$$
\begin{aligned}
S_{k, K}(p) & =\operatorname{det}\left(D \phi_{K}\right) \int_{0}^{1} \int_{0}^{1-\zeta} \int_{0}^{1-\zeta}(1-\zeta)^{c-a-b} \xi^{a} \eta^{b} \mathrm{~d} \xi \mathrm{d} \eta \mathrm{d} \zeta \\
& =\operatorname{det}\left(D \phi_{K}\right) \int_{\hat{K}} \hat{p}(\xi, \eta, \zeta) \mathrm{d} \hat{x}=\int_{K} p \mathrm{~d} x
\end{aligned}
$$

We can now examine the behaviour of our quadrature scheme on the kinds of products arising in a typical weak formulation. In fact, we will only need Lemma 5.1 to be true for $\tilde{f} \in Q_{2 k}^{2 k, 2 k, 2 k}$, which is a subspace of $Q_{2 k+1}^{2 k+1,2 k+1,2 k+1}$ 
Theorem 5.2. Let $K$ be an affine pyramid; fix $k \geq 1$; let $s \in\{0,1,2,3\}$ and let $A \in \Theta^{s}(K)$ be a constant tensor field. Then for any $u, v \in \mathcal{U}_{k}^{(s)}(K)$, the quadrature scheme $S_{k, K}$ is exact for the 0-form $A(u, v)$, i.e.

$$
S_{k, K}\left(A^{i j} u_{i} v_{j}\right)=\int_{K} A^{i j} u_{i} v_{j} \mathrm{~d} x
$$

Proof of Theorem 5.2. Let $u, v \in \mathcal{R}_{k}^{(s)}(K) . A \in \Theta^{(s)}(K)$ is a constant and so, by the first part of Lemma 4.3, in infinite reference coordinates, the function $A(u, v)$ satisfies:

$$
A^{\tilde{i} \tilde{j}} u_{\tilde{j}} v_{\tilde{i}} \in Q_{2 k}^{2 k, 2 k, 2 k}
$$

Hence by Lemma 5.1,

$$
S_{k, K}\left(A^{i j} u_{j} v_{i}\right)=\int_{K} A^{i j} u_{j} v_{i} .
$$

Observe that for the spaces $\mathcal{U}_{k}^{(3)}(K)$, the integrand, $A^{\tilde{i} \tilde{j}} u_{\tilde{j}} v_{\tilde{i}} \in Q_{2 k-2}^{2 k-2,2 k-2,2 k-2}$, so we could in fact use the scheme $S_{k-1, K}$.

\subsection{Consistency error estimates}

Having constructed approximation spaces which satisfy the hypotheses of Theorem 2.5, and demonstrated a quadrature rule which is exact for products of basis functions, the final step is to establish an estimate of the quadrature-related consistency error for each of the spaces in the decompositions in terms of the reference norms. We will use the quadrature scheme, $S_{k, K}(\cdot)$ and associated error functional $E_{k, K}(\cdot)$ introduced in Section 5.1. We will also use the pointwise representation, $A(u, v)=A^{i j} u_{i} v_{j}$ given in (3.2).

Lemma 5.3. For any $s \in\{0,1,2,3\}$ and an affine pyramid, $K$, let $v \in \mathcal{X}_{r, k}^{(s)}(K), w \in \mathcal{R}_{k}^{(s)}(K)$ and $A \in W^{k+1, \infty} \Theta^{(s)}(K)$. Then the quadrature error in the evaluation of $\int_{K} A(u, v)=\int_{K} A^{i j} u_{i} v_{j}$ using the scheme $S_{k, K}(\cdot)$ can be bounded in terms of the reference (semi-)norms

$$
\left|E_{k, K}(A(v, w))\right| \leq C \operatorname{det}\left(D \phi_{K}\right)|A|_{k-r+1, \infty, \hat{K}}|\hat{v}|_{r, \hat{K}}\|\hat{w}\|_{0, \hat{K}}
$$

where $C=C(k)$ is a constant that depends only on $k$.

Proof. We can transform the error functional onto the reference pyramid using (5.4).

$$
E_{k, K}(A(v, w))=E_{k, K}\left(A^{i j} v_{i} w_{j}\right)=E_{k, \hat{K}}\left(\operatorname{det}\left(D \phi_{K}\right) A^{\hat{\imath} \hat{j}} v_{\hat{i}} w_{\hat{j}}\right)=\operatorname{det}\left(D \phi_{K}\right) E_{k, \hat{K}}\left(A^{\hat{\imath} \hat{j}} v_{\hat{i}} w_{\hat{j}}\right) .
$$

We are able to take $\operatorname{det}\left(D \phi_{K}\right)$ outside the integral because $\phi_{K}$ is affine. Define the linear functional $G \in W^{k-r+1, \infty} \Theta^{(s)}(\hat{K})^{\prime}$ as

$$
G(B)=E_{k, \hat{K}}\left(B^{\hat{\imath} \hat{j}} v_{\hat{i}} w_{\hat{j}}\right) \quad \forall B \in W^{k-r+1, \infty} \Theta^{(s)}(\hat{K}) .
$$

Since $S_{k}(\cdot)$ takes point values of its argument,

$$
|G(B)| \leq C\left\|B^{\hat{i} \hat{j}} v_{\hat{i}} w_{\hat{j}}\right\|_{\infty, \hat{K}} \leq C\|B\|_{k-r+1, \infty, \hat{K}}\|\hat{v}\|_{\infty, \hat{K}}\|\hat{w}\|_{\infty, \hat{K}}
$$

Furthermore, all norms are equivalent on the finite dimensional spaces, $\mathcal{X}_{r, k}^{(s)}(\hat{K})$ and $\mathcal{R}_{k}^{(s)}(\hat{K})$, and, by the last part of Lemma $4.9,|\cdot|_{r, \hat{K}}$ is a norm for $\mathcal{X}_{r, k}^{(s)}$. So $G$ is continuous and $\|G\| \leq C|\hat{v}|_{r, \hat{K}}\|\hat{w}\|_{0, \hat{K}}$. All of the 
equivalences of norms are done on the reference pyramid, so the constant, $C$ depends only on $k$ (in particular, it does not depend on $K$ ).

From the definition of $\mathcal{X}_{r, k}^{(s)}$, we know that each $v_{\hat{i}} \circ \phi \in Q_{r}^{r+1, r+1,0}$ and by Lemma $4.3, w_{\hat{j}} \circ \phi \in Q_{k}^{k, k, k}$ for each $\hat{j} \in\left\{1, \ldots,\left(\begin{array}{l}3 \\ s\end{array}\right)\right\}$. Now suppose that $B$ is polynomial of degree $k-r$, i.e. each component, $B^{\hat{i} \hat{j}} \in P^{k-r}$ for each $\hat{i}, \hat{j} \in\left\{1, \ldots,\left(\begin{array}{l}3 \\ s\end{array}\right)\right\}$. Then $B^{\hat{i} \hat{j}} \circ \phi \in Q_{k-r}^{[k-r, k-r]}$. We can assemble these facts to see that

$$
\left(B^{\hat{i} \hat{j}} v_{\hat{i}} w_{\hat{j}}\right) \circ \phi=\left(B^{\hat{i} \hat{j}} \circ \phi\right)\left(v_{\hat{i}} \circ \phi\right)\left(w_{\hat{j}} \circ \phi\right) \in Q_{2 k+1}^{2 k+1,2 k+1,2 k+1} .
$$

So, by Lemma 5.1, the quadrature error, $E_{k, \hat{K}}\left(B^{\hat{i} \hat{j}} v_{\hat{i}} w_{\hat{j}}\right)=0$. Therefore, $P^{k-r} \subset$ ker $G$ and we can apply Theorem 2.2 (the Bramble-Hilbert Lemma) to obtain

$$
|G(A)| \leq C|A|_{k-r+1, \infty, \hat{K}}|v|_{r, \hat{K}}\|w\|_{0, \hat{K}} \quad \forall A \in W^{k-r+1, \infty} \Theta^{(s)}(\hat{K}) .
$$

For some constant $C=C(k)$. Substituting (5.7) and (5.6) gives the desired result.

We can now apply a scaling argument to get an element-wise estimate on the quadrature error. Recall that we defined the interpolation operator, $\Phi_{K}^{(s)}: \mathcal{H}^{(s), 1 / 2+\epsilon}(K) \rightarrow \mathcal{R}_{k}^{(s)}(K)$ in (A.9).

Lemma 5.4. Let $K$ be an affine pyramid satisfying the shape-regularity condition, (3.8), for some $\rho \geq 1$. Fix $s \in\{0,1,2,3\}$ and take $k \geq 2$. Then

$$
\begin{aligned}
& \forall u \in H^{k} \Lambda^{(s)}(K), w \in \mathcal{R}_{k}^{(s)}(K) \text { and } A \in W^{k+1, \infty} \Theta^{(s)}(K) \\
& \left.\left|E_{k, K}\left(A\left(\Phi_{k, K}^{(s)} u, w\right)\right)\right| \leq\left(C h^{k+1}\right)\right)\|A\|_{k+1, \infty, K}\|u\|_{k, K}\|w\|_{0, K}
\end{aligned}
$$

where $C=C(k)$ a constant dependent only on $k$, and $0<h<C$.

Proof. Use the decomposition given in Theorem 4.10 to write

$$
\Phi_{k, K}^{(s)} u=v_{0}+\cdots+v_{k} \text { where } v_{r} \in \mathcal{X}_{r, k}^{(s)}(K) .
$$

By Lemma 5.3, we know that for each $r \in\{0 \ldots k\}$,

$$
\left|E_{k, K}\left(A\left(v_{r}, w\right)\right)\right| \leq C\left|D \phi_{K}\right||A|_{k-r+1, \infty, \hat{K}}\left|v_{r}\right|_{r, \hat{K}}\|w\|_{0, \hat{K}}
$$

The interpolation operator is bounded on $\mathcal{H}^{(s), 1 / 2+\epsilon}(K)$ which is a subset of $H^{3 / 2+\epsilon} \Lambda^{(s)}(K)$ so Theorem 2.5 is applicable with $\alpha>3 / 2$. Pick some $\alpha \in(3 / 2,2]$ so that when $r \geq 2$ we can use the first estimate, (2.11), to obtain:

$$
\left|E_{k, K}\left(A\left(v_{r}, w\right)\right)\right| \leq C\left|D \phi_{K}\right||A|_{k-r+1, \infty, \hat{K}}|u|_{r, \hat{K}}\|\hat{w}\|_{0, \hat{K}}
$$

Now apply the inequalities (3.9) and (3.10) to the semi-norms (and norm) on the right-hand side to obtain

$$
\begin{aligned}
\left|E_{k, K}\left(A\left(v_{r}, w\right)\right)\right| & \leq C\left|D \phi_{K}\right| h^{k-r+1-2 s} \rho^{2 s}|A|_{k-r+1, \infty, K} \frac{h^{r+s}}{\left|D \phi_{K}\right|^{1 / 2}}|u|_{r, K} \frac{h^{s}}{\left|D \phi_{K}\right|^{1 / 2}}\|\hat{w}\|_{0, K} \\
& =C h^{k+1}|A|_{k-r+1, \infty, K}|u|_{r, K}\|w\|_{0, K}
\end{aligned}
$$

where the generic constant, $C$ still depends only on $k$. 
When $r=1$, we can similarly apply the second estimate from Theorem 2.5 given in (2.12) to obtain:

$$
\left|E_{k, K}\left(A\left(v_{1}, w\right)\right)\right| \leq C h^{k+1}|A|_{k, \infty, K}\left(|u|_{1, K}+h|u|_{2, K}\right)\|w\|_{0, K}
$$

For $r=0$, note that $\left\|v_{0}\right\|_{0, \hat{K}} \leq C\|u\|_{3 / 2+\epsilon, \hat{K}} \leq C\left(|u|_{0, \hat{K}}+|u|_{1, K}+|u|_{2, \hat{K}}\right)$, so

$$
\left|E_{k, K}\left(A\left(v_{0}, w\right)\right)\right| \leq C h^{k+1}|A|_{k+1, \infty, K}\left(|u|_{0, K}+h|u|_{1, K}+h^{2}|u|_{2, K}\right)\|w\|_{0, K}
$$

Summing over the $v_{r}$, we obtain (5.10).

Summing these errors over each element gives an estimate for the global consistency error due to the numerical integration (we shall ignore the $O\left(h^{k+2}\right)$ terms). Recall that in (4.8) we defined the global approximation space, $\mathcal{S}_{h}^{(s)} \subset \mathcal{H}^{(s)}(\Omega)$

Theorem 5.5. Let $s \in\{0,1,2,3\}, k \geq 2$ and assume that $\mathcal{S}_{h}^{(s)}$ is constructed using a shape regular mesh, $\mathcal{T}_{h}$ and finite elements, $\mathcal{R}_{k}^{(s)}(K)$ for each $K \in \mathcal{T}_{h}$. Let $A \in W^{k+1, \infty} \Theta^{(s)}(\Omega)$ and $u \in H^{(s)} \Lambda^{k}(\Omega$. Then the interpolant $\Phi_{h}^{(s)} u \in \mathcal{S}_{h}^{(s)}$ satisifies

$$
\begin{aligned}
& \sup _{w_{h} \in \mathcal{S}_{h}^{(s)}} \frac{\left|\int_{\Omega} A\left(\Phi_{h}^{(s)} u, w_{h}\right)-\sum_{K \in \mathcal{T}_{h}} S_{K, k}\left(A\left(\Phi^{(s)} u, w_{h}\right)\right)\right|}{\left\|w_{h}\right\|_{0}} \leq C h^{k+1}\|A\|_{k+1, \infty, \Omega}\|u\|_{k, \Omega} \\
& \sup _{w_{h} \in \mathcal{S}_{h}^{(s)},\left\|w_{h}\right\|_{0}=1}\left|\int_{\Omega} A\left(\Phi_{h}^{(s)} u, w_{h}\right)-\sum_{K \in \mathcal{T}_{h}} S_{K, k}\left(A\left(\Phi^{(s)} u, w_{h}\right)\right)\right| \leq C h^{k+1}\|A\|_{k+1, \infty, \Omega}\|u\|_{k, \Omega} .
\end{aligned}
$$

Here $C>0$ is a constant which only depends on $k$, and $0<h<C$.

Proof. Let $w_{h} \in \mathcal{S}_{h}^{(s)}$.

$$
\begin{aligned}
\left|\int_{\Omega} A\left(\Phi_{h}^{(s)} u, w_{h}\right)-\sum_{K \in \mathcal{T}_{h}} S_{K, k}\left(A\left(\Phi^{(s)} u, w_{h}\right)\right)\right| & \leq C \sum_{K \in \mathcal{T}_{h}} E_{k, K}\left(A\left(\Phi_{k, K}^{(s)} u, w_{h}\right)\right) \\
& \leq C h^{k+1} \sum_{K \in \mathcal{T}_{h}}\|A\|_{k+1, \infty, K}\|u\|_{k, K}\left\|w_{h}\right\|_{0, K} \\
& \leq C h^{k+1}\|A\|_{k+1, \infty, \Omega}\left(\sum_{K \in \mathcal{T}_{h}}\|u\|_{k, K}^{2}\right)^{1 / 2}\left(\sum_{K \in \mathcal{T}_{h}}\left\|w_{h}\right\|_{0, K}^{2}\right)^{1 / 2} \\
& \leq C h^{k+1}\|A\|_{k+1, \infty, \Omega}\|u\|_{k, \Omega}\left\|w_{h}\right\|_{0, \Omega}
\end{aligned}
$$

Dividing through by $\left\|w_{h}\right\|_{0, \Omega}$ gives the result.

Note that as with the classical theory, the error estimates decay like $O\left(h^{k+1}\right)$ but these are emphatically not $h p$-estimates. The degree, $k$, enters into the constants in several places, which is to be expected from arguments that rely on the Bramble-Hilbert Lemma. 


\section{Conclusion}

The conventional finite element wisdom is that a $k$ th order method requires a $k$ th order quadrature scheme. More specifically, the conventional wisdom is that the quadrature scheme must be able to integrate products of shape functions and of their exterior derivatives exactly. We have shown that this is still true for a family of high order pyramidal finite elements, but that the non-polynomial nature of pyramidal elements requires some unconventional reasoning to justify the wisdom.

We finish with a few observations.

\subsection{The new elements}

The description of the family of high order finite elements for the de Rham complex that we introduced is new. We will examine these elements in more detail in future work, but a couple of notes are worth recording here.

- The approximation spaces for the first family in the sequence, $\mathcal{R}_{k}^{(0)}(K)$ are the same as Zaglmayr's elements, as described in [9], and which [3] describes as optimal with respect to their dimension and compatibility with neighbouring elements.

- Lemma 4.4 shows that the $\mathcal{R}_{k}^{(s)}(K)$ spaces contain polynomials corresponding to the tetrahedron of the first type. Zaglmayr has constructed pyramidal elements containing polynomials corresponding to both types of tetrahedron, but only those corresponding to the second type are presented in [9]. It would, clearly, be interesting to compare our $\mathcal{R}_{k}^{(s)}(K)$ spaces with the construction for the first type.

\subsection{The original elements}

From Lemma 4.6, we know that $\mathcal{R}_{k}^{(s)}(K) \subseteq \mathcal{U}_{k}^{(s)}(K)$ and hence the interpolant $\Phi_{h}^{(s)} u$ also lives in the original finite element space based on $\mathcal{U}_{k}^{(s)}(K)$. The important property of $w$ used in Lemma 5.3 , was that $w_{\hat{j}} \circ \phi \in Q_{k}^{k, k, k}$, which was proved in Lemma 4.3. In fact, Lemma 4.3 is still true when $\mathcal{R}_{k}^{(s)}(K)$ is replaced by $\mathcal{U}_{k}^{(s)}(K)$. Hence we could take $w \in \mathcal{U}_{k}^{(s)}(K)$ in Lemmas 5.3 and 5.4 and derive a version of Theorem 5.5 that gives a consistency error approximation for finite elements based on the original spaces.

\subsection{General bilinear forms}

The error estimate may be applied to more general bilinear forms because of the commutativity $d \circ \Pi_{h}^{(s)}=$ $\Pi_{h}^{(s+1)} \circ d$. For example, the consistency error for the elliptic bilinear form, $(2.3)$, is

$$
\begin{aligned}
\sup _{v \in \mathcal{S}_{h}^{(0)}} \frac{\left|a\left(\Phi_{h}^{(0)} u, v\right)-a_{h}\left(\Phi_{h}^{(0)} u, v\right)\right|}{\|v\|_{1}} & \leq \sup _{v \in \mathcal{S}_{h}^{(0)},\|\mathrm{d} v\|_{0}=1} \int_{\Omega} A\left(d \Phi_{h}^{(0)} u, \mathrm{~d} v\right)-\sum_{K \in \mathcal{T}_{h}} S_{K, k}\left(A\left(d \Phi_{h}^{(0)} u, \mathrm{~d} v\right)\right) \\
& \leq \sup _{w \in \mathcal{S}_{h}^{(1)},\|w\|_{0}=1} \int_{\Omega} A\left(\Phi_{h}^{(1)} \mathrm{d} u, w\right)-\sum_{K \in \mathcal{T}_{h}} S_{K, k}\left(A\left(\Phi_{h}^{(1)} \mathrm{d} u, w\right)\right) \\
& \leq C h^{k+1}\|A\|_{k+1, \infty, \Omega}\|\mathrm{d} u\|_{k, \Omega} \\
& <C h^{k+1}\|A\|_{k+1, \infty, \Omega}\|u\|_{k+1, \Omega} .
\end{aligned}
$$

\section{Appendix A. Properties of the new approximation spaces, $\mathcal{R}_{k}^{(s)}$}

In this appendix, we have collected proofs of various Lemmas in that describe the properties of the approximation spaces $\mathcal{R}_{k}^{(s)}$ which were introduced in Section 4. We also prove Theorem 4.10, which is the key result required to obtain the main result of this paper. We begin, however, by briefly recalling the approximation spaces $\mathcal{U}_{k}^{(s)}(K)$ for the finite elements presented in [17]. 
We remind the reader that the goal in [17] was to construct a family of high-order conforming finite elements which were compatible through the appropriate boundary traces with data from neighbouring tetrahedral and hexahedral elements. The trace spaces for the tetrahedral Lagrange, Nedelec edge and Nedelec face elements are given in [15], and are polynomial in nature. Our construction proceeded by first constructing underlying approximation spaces $\overline{\mathcal{U}}_{k}^{(s)}$ defined for each order, $k \geq 1$, and then identifying subspaces $\mathcal{U}_{k}^{(s)}(K)$ whose members have the correct exterior traces.

- $H^{1}$-conforming element underlying space:

$$
\overline{\mathcal{U}}_{k}^{(0)}=Q_{k}^{k, k, k-1} \oplus \operatorname{span}\left\{\frac{z^{k}}{(1+z)^{k}}\right\} .
$$

- $H($ curl)-conforming element underlying space:

$$
\overline{\mathcal{U}}_{k}^{(1)}=Q_{k+1}^{k-1, k, k-1} \times Q_{k+1}^{k, k-1, k-1} \times Q_{k+1}^{k, k, k-2} \oplus\left\{\frac{z^{k-1}}{(1+z)^{k+1}}\left(\begin{array}{c}
z \frac{\partial r}{\partial x} \\
z \frac{\partial r}{\partial y} \\
-r
\end{array}\right), \quad r \in Q^{k, k}[x, y]\right\} .
$$

- $H($ div)-conforming element space:

$$
\begin{aligned}
\overline{\mathcal{U}}_{k}^{(2)}= & Q_{k+2}^{k, k-1, k-2} \times Q_{k+2}^{k-1, k, k-2} \times Q_{k+2}^{k-1, k-1, k-1} \\
& \oplus \frac{z^{k-1}}{(1+z)^{k+2}}\left(\begin{array}{c}
0 \\
2 s \\
s_{y}(1+z)
\end{array}\right) \oplus \frac{z^{k-1}}{(1+z)^{k+2}}\left(\begin{array}{c}
2 t \\
0 \\
t_{x}(1+z)
\end{array}\right),
\end{aligned}
$$

where $s(x, y) \in Q^{k-1, k}[x, y], \quad t(x, y) \in Q^{k, k-1}[x, y]$.

- $L^{2}$-conforming element underlying space:

$$
\mathcal{U}_{k}^{(3)}=Q_{k+3}^{k-1, k-1, k-1}
$$

For an element defined on a pyramid, $K$, the underlying approximation space, $\overline{\mathcal{U}}_{k}^{(s)}(K)$ is defined as the space containing all the $s$-forms whose components induced by the infinite pyramid coordinate system lie in $\overline{\mathcal{U}}_{k}^{(s)}::^{6}$

$$
\overline{\mathcal{U}}_{k}^{(s)}(K)=\left\{u \in \Lambda^{(s)}(K):\left(u_{\tilde{i}}\right)_{\tilde{i} \in\left\{1, \ldots,\left(\begin{array}{l}
3 \\
s
\end{array}\right)\right\}} \in \overline{\mathcal{U}}_{k}^{(s)}\right\}
$$

By inspection, it can be seen that the exterior derivative $d: \overline{\mathcal{U}}_{k}^{(s)} \rightarrow \overline{\mathcal{U}}_{k}^{(s+1)}$ is well defined, and so, since $d$ is independent of coordinates, the exterior derivative on the spaces on each element,

$$
d: \overline{\mathcal{U}}_{k}^{(s)}(K) \rightarrow \overline{\mathcal{U}}_{k}^{(s+1)}(K)
$$

is also well defined.

In order to construct pyramidal elements that are compatible with neighbouring tetrahedral (and hence polynomial) elements, subspaces of the underlying approximation spaces, $\overline{\mathcal{U}}_{k}^{(s)}(K)$ are identified that contain

\footnotetext{
${ }^{6}$ We are using coordinate transformations here, but in [17], we used the equivalent approach of defining the underlying spaces as the pullbacks, $\overline{\mathcal{U}}_{k}^{(s)}(\hat{K})=\left\{\left(\phi^{-1}\right)^{*} v: v \in \overline{\mathcal{U}}_{k}^{(s)}\right\}$ and $\overline{\mathcal{U}}_{k}^{(s)}(K)=\left\{\phi_{K}^{*} v: v \in \overline{\mathcal{U}}_{k}^{(s)}(\hat{K})\right\}$.
} 
only those functions whose traces on the triangular faces of the pyramid are contained in the trace space of the corresponding tetrahedral element. By construction, the traces of the underlying spaces on the quadrilateral face of the pyramid already match those of the corresponding hexahedral elements. Upon enforcing these constraints, the approximation spaces obtained are denoted $\mathcal{U}_{k}^{(s)}(K)$. We shall denote by $\mathcal{U}_{k, 0}^{(s)}(K)$ the subspaces of $\mathcal{U}_{k}^{(s)}(K)$ with zero boundary traces.

Each approximation space $\mathcal{U}_{k}^{(s)}(K)$ is equipped with a set of degrees of freedom, $\Sigma^{(s)}(K)$. These included (exterior) degrees of freedom which are identical to those of neighbouring tetrahedral and hexahedral elements, and projection-based degrees of freedom. In turn, $\Sigma^{(s)}(K)$ induces a linear interpolation operator,

$$
\Pi_{k, K}^{(s)}: H^{2} \Lambda^{(s)}(K) \rightarrow \mathcal{U}_{k}^{(s)}(K), \quad \text { so that } m(u)=m\left(\Pi_{k, K}^{(s)} u\right) \quad \forall m \in \Sigma^{(s)}(K)
$$

which completes the definition of the finite elements. The regularity assumption is not optimal, and can perhaps be relaxed; it should allow for point evaluations at the vertices of the pyramid for the $s=0$ elements. In [8], the authors specify the optimal regularity needed for the projection-based interpolants of, we anticipate a similar result holds for the more explicit construction given in [17].

We now examine the properties of the new approximation spaces, $\mathcal{R}_{k}^{(s)}$ introduced in Section 4.

Proof of Lemma 4.4. Since the set of polynomials $P^{k}$ is invariant under affine transformation, we can work in the reference coordinate system, $\hat{x}$. Recall the components of the proxy representation of some $u \in \Lambda^{(s)}(K)$ in this coordinate system are denoted $\hat{u}=\left(u_{\hat{i}}\right)$, where $1 \leq \hat{i} \leq\left(\begin{array}{l}3 \\ s\end{array}\right)$. We will need to show that if all the components, $u_{\hat{i}} \in P^{k-1}$ for $s=1,2,3$ (or, for $s=0, u_{\hat{i}} \in P^{k}$ ) then $u \in \mathcal{R}_{k}^{(s)}(K)$. Equivalently, we need to show $\tilde{u} \in \mathcal{R}_{k}^{(s)}$. We use the transformation rule, (3.5), along with the explicit weights associated with the coordinate change $\phi: K_{\infty} \rightarrow \hat{K}$ given in $(3.12 \mathrm{a})-(3.12 \mathrm{~d})$.

First, consider the case when $s=0$. Let $u \in \Lambda^{(0)}(K)$ be a monomial with reference coordinate representation $\hat{u}(\xi, \eta, \zeta)=\xi^{a} \eta^{b}(1-\zeta)^{c}$ where $a+b+c \leq k$. Then

$$
\tilde{u}=\left(w_{\phi}^{(0)}\right)^{-1} \hat{u} \circ \phi=\frac{x^{a} y^{b}}{(1+z)^{a+b+c}} \in Q_{k}^{[k, k]}=\mathcal{R}_{k}^{(0)}
$$

Similarly, for $s=3$, take $u \in \Lambda^{(3)}(K)$ as $\hat{u}(\xi, \eta, \zeta)=\xi^{a} \eta^{b}(1-\zeta)^{c}$ for $a+b+c \leq k-1$. Then

$$
\tilde{u}=\frac{x^{a} y^{b}}{(1+z)^{a+b+c+4}} \in Q_{k+3}^{[k-1, k-1]}=\mathcal{R}_{k}^{(3)} .
$$

The $s=1$ case involves a little more work. Let $u \in \Lambda^{(1)}(K)$ have polynomial components, $u_{\hat{i}} \in P^{k-1}$. We can find $q \in \Lambda^{(0)}(K)$ with representation $\hat{q} \in P^{k}$ such that $v:=u-\nabla q=\left(v_{\hat{1}}, v_{\hat{2}}, 0\right)$. By the result for $s=0$, $q \in \mathcal{R}_{k}^{(0)}(K)$, and so (by $\left.(4.3 \mathrm{~b})\right) \nabla q \in \mathcal{R}_{k}^{(1)}(K)$. We need to show that $v \in \mathcal{R}_{k}^{(1)}(K)$. Both $v_{\hat{1}}$ and $v_{\hat{2}}$ are in $P^{k-1}$. Suppose first that $v_{\hat{1}}=\xi^{a} \eta^{b}(1-\zeta)^{c}$ where $m:=a+b+c \leq k-1$ and $v_{\hat{2}}=0$.

$$
\begin{aligned}
\tilde{v}=\left(w_{\phi}^{(1)}\right)^{-1} \hat{v} \circ \phi & =\frac{1}{(1+z)^{2}}\left(\begin{array}{ccc}
1+z & 0 & 0 \\
0 & 1+z & 0 \\
-x & -y & 1
\end{array}\right)\left(\begin{array}{c}
\frac{x^{a} y^{b}}{(1+z)^{m}} \\
0 \\
0
\end{array}\right) \\
& =\left(\begin{array}{c}
\frac{x^{a} y^{b}}{(1+z)^{m+1}} \\
0 \\
-\frac{x^{a+1} y^{b}}{(1+z)^{m+2}}
\end{array}\right)=\frac{1}{(1+z)^{m+1}}\left(\begin{array}{c}
\left(1-\frac{a+1}{m+1}\right) x^{a} y^{b} \\
-\frac{b}{m+1} x^{a+1} y^{b-1} \\
0
\end{array}\right)+\frac{1}{m+1} \nabla \frac{x^{a+1} y^{b}}{(1+z)^{m+1}} .
\end{aligned}
$$


We compare this last expression with the definition, (4.3b), to verify that $\tilde{v} \in \mathcal{R}_{k}^{(1)}$. Note that when $a=m$ (which includes the case $a=k-1$ ), the first term vanishes, because $b=0$ and $1-\frac{a+1}{m+1}=0$. That is, $\left(\xi^{a}, 0,0\right)^{t}$ is an exact 1-form. An identical calculation establishes the same result when $v_{\hat{1}}=0$ and $v_{\hat{2}}=\xi^{a} \eta^{b}(1-\zeta)^{c}$.

For $s=2$, the change of coordinates formula for $u \in \Lambda^{(2)}(K)$ is

$$
\tilde{u}=\left(w_{\phi}^{(2)}\right)^{-1} \hat{u} \circ \phi=\frac{1}{(1+z)^{3}}\left(\begin{array}{ccc}
1 & 0 & x \\
0 & 1 & y \\
0 & 0 & 1+z
\end{array}\right)\left(\begin{array}{l}
u_{\hat{\hat{1}}} \\
u_{\hat{2}} \\
u_{\hat{3}}
\end{array}\right) \circ \phi .
$$

Suppose that $u_{\hat{1}}=\xi^{a} \eta^{b}(1-\zeta)^{c}$ with $m:=a+b+c \leq k-1$. Apply (A.5) to see that the contribution to $\tilde{u}$ is $\left(\frac{x^{a} y^{b}}{(1+z)^{m+3}}, 0,0\right)^{t}$. Let $p=\frac{1}{m+2} \frac{x^{a} y^{b}}{(1+z)^{m+2}} \in Q_{k+1}^{[k-1, k-1]}$ and observe that $\frac{x^{a} y^{b}}{(1+z)^{m+3}}=-\frac{\partial p}{\partial z}$ and $\frac{\partial p}{\partial x}=$ $\frac{a}{m+2} \frac{x^{a-1} y^{b}}{(1+z)^{m+2}} \in Q_{k+2}^{[k-1, k-1]}$ (the case $b=m$ implies that $a=0$ and therefore $\frac{\partial p}{\partial x}=0$ ). Hence

$$
\left(w_{\phi}^{(2)}\right)^{-1}\left(\begin{array}{c}
\xi^{a} \eta^{b}(1-\zeta)^{c} \\
0 \\
0
\end{array}\right) \circ \phi=\nabla \times\left(\begin{array}{l}
0 \\
p \\
0
\end{array}\right)-\left(\begin{array}{c}
0 \\
0 \\
\frac{\partial p}{\partial x}
\end{array}\right) \in \mathcal{R}_{k}^{(2)} .
$$

Polynomials in the second component $u_{\hat{2}}$ can be dealt with similarly. When $u_{\hat{3}}=\xi^{a} \eta^{b}(1-\zeta)^{c}$, the contribution to $\tilde{u}$ is $\left(\frac{x^{a+1} y^{b}}{(1+z)^{m+3}}, \frac{x^{a} y^{b+1}}{(1+z)^{m+3}}, \frac{x^{a} y^{b}}{(1+z)^{m+2}}\right)^{t}$. Hence

$$
\left(w_{\phi}^{(2)}\right)^{-1}\left(\begin{array}{c}
0 \\
0 \\
\xi^{a} \eta^{b}(1-\zeta)^{c}
\end{array}\right) \circ \phi=\nabla \times \frac{1}{m+2}\left(\begin{array}{c}
-\frac{x^{a} y^{b+1}}{(1+z)^{m+2}} \\
\frac{x^{a+1} y^{b}}{(1+z)^{m+2}} \\
0
\end{array}\right)+\left(\begin{array}{c}
0 \\
0 \\
\left(1-\frac{a+b+2}{m+2}\right) \frac{x^{a} y^{b}}{(1+z)^{m+2}}
\end{array}\right) \in \mathcal{R}_{k}^{(2)}
$$

Note that $\frac{x^{a} y^{b}}{(1+z)^{m+2}} \in Q_{k}^{[k-1, k-1]}$ unless $a=m$ or $b=m$, but in these cases, $\left(1-\frac{a+b+2}{m+2}\right)=0 .{ }^{7}$ Hence $\tilde{u} \in \mathcal{R}_{k}^{(2)}$, proving the assertion.

Proof of Lemma 4.5. An alternative, but less self-contained, way of stating this lemma would be to claim that the trace spaces of the $\mathcal{R}_{k}^{(s)}(K)$ elements are identical to those of the original $\mathcal{U}_{k}^{(s)}(K)$ elements, and are hence compatible through their exterior traces with neighboring elements. Consequently, the strategy and tools from [17] may be reapplied in an identical fashion. We will therefore just provide a sketch of how this may be done.

We must demonstrate that the appropriate exterior traces of the $\mathcal{R}_{k}^{(s)}(K)$ functions lie in the trace spaces of the corresponding tetrahedral or hexahedal approximation spaces. Secondly, we need to show that if a function has a valid (polynomial) trace on the boundary of the pyramid, then it can be represented by some member of $\mathcal{R}_{k}^{(s)}(K)$.

For the first step, convenient definitions of the tetrahedral and hexahedral spaces may be found in [15] and the traces of these spaces are identified explicitly in [17]. It is just a matter of exhaustive checking to determine that the inclusion holds. As an illustration, observe that members of the $\mathcal{R}_{k}^{(0)}$ which are non-zero on the face $y=0$ of the infinite pyramid can be expressed in terms of monomials $\frac{x^{a}}{(1+z)^{c}}$, where $a+c \leq k$, which map to $\xi^{a} \zeta^{k-a-c}$. These will span all polynomials of degree $k$ on the face $\eta=0$ of the finite pyramid, which is precisely the trace space of the $k$ th order Lagrange tetrahedron.

The second step is equivalent to requiring that the combined external degrees of freedom inherited from the tetrahedra and hexahedra across all the vertices, edges and faces of the pyramid be dual to the trace spaces

\footnotetext{
${ }^{7}$ This is the observation that $\left(0,0, \xi^{a}\right)^{t}$ and $\left(0,0, \eta^{b}\right)^{t}$ are exact 2 -forms.
} 
on the pyramid. This can be proved by demonstrating a linearly independent set of pyramidal shape functions with non-zero traces that is the same size as the set of external degrees of freedom. This task can be made more manageable by instead showing that it is possible to achieve the lowest order bubble on each face, edge and vertex of the pyramid that is zero on every other face, edge or vertex, respectively. (N.B. For completeness we actually presented an example of all the bubbles in the appendix of [17], not just the lowest order). Happily, the shape functions associated with the external degrees of freedom presented for the $\mathcal{U}_{k}^{(s)}(K)$ in Tables 2-4 of [17] also suffice for the $\mathcal{R}_{k}^{(s)}(K)$.

Note that the original approximation spaces, $\mathcal{U}_{k}^{(s)}(K)$ were defined by explicitly identifying the subsets of underlying spaces, $\overline{\mathcal{U}}_{k}^{(s)}(K)$ which had such polynomial trace spaces. For the $\mathcal{R}_{k}^{(s)}(K)$, the polynomial trace property is inherent and this additional step is not required.

Proof of Lemma 4.6. We must first establish the inclusions

$$
\mathcal{R}_{k}^{(s)} \subseteq \overline{\mathcal{U}}_{k}^{(s)} \quad \forall s \in\{0,1,2,3\}
$$

The inclusions

$$
Q_{n}^{[l, m]} \subseteq\left(Q_{n}^{l, m, \min \{l, m\}-1}+Q_{n}^{0,0, \min \{l, m\}}\right) \subseteq Q_{n}^{l, m, \min \{l, m\}} .
$$

can be verified from the definition, (4.1). By the first inclusion, $Q_{k}^{[k, k]} \subseteq Q_{k}^{k, k, k-1}+Q_{k}^{0,0, k}$, which gives the $s=0$ case: $\mathcal{R}_{k}^{(0)} \subseteq \overline{\mathcal{U}}_{k}^{(0)}$.

The $s=0$ result implies $\nabla \mathcal{R}_{k}^{(0)} \subseteq \nabla \overline{\mathcal{U}}_{k}^{(0)}$. For $s=1$, first recall that $\mathcal{R}_{k}^{(1)}=\left(Q_{k+1}^{[k-1, k]} \times Q_{k+1}^{[k, k-1]} \times\{0\}\right) \oplus$ $\left\{\nabla u: u \in Q_{k}^{[k, k]}\right\}$. Since $\nabla \overline{\mathcal{U}}_{k}^{(0)} \subset \overline{\mathcal{U}}_{k}^{(1)}$, we have $\nabla Q_{k}^{[k, k]} \subset \overline{\mathcal{U}}_{k}^{(1)}$. Additionally, apply (A.6) and the definition of $\overline{\mathcal{U}}_{k}^{(1)}$ given in (A.1b), to obtain

$$
\left(Q_{k+1}^{[k-1, k]} \times Q_{k+1}^{[k, k-1]} \times\{0\}\right) \subseteq\left(Q_{k+1}^{k-1, k, k-1} \times Q_{k+1}^{k, k-1, k-1} \times\{0\}\right) \subset \overline{\mathcal{U}}_{k}^{(1)} .
$$

This establishes that $\mathcal{R}_{k}^{(1)} \subseteq \overline{\mathcal{U}}_{k}^{(1)}$.

The $s=2$ case may be proved similarly. Again, first recall $\mathcal{R}_{k}^{(2)}=\left(\{0\} \times\{0\} \times Q_{k+2}^{[k-1, k-1]}\right) \oplus\{\nabla \times u: u$ $\left.\in\left(Q_{k+1}^{[k-1, k]} \times Q_{k+1}^{[k, k-1]} \times\{0\}\right)\right\}$. We also have that $\nabla \times \overline{\mathcal{U}}_{k}^{(1)} \subset \overline{\mathcal{U}}_{k}^{(2)}$. Using the result for $s=1$, therefore, we see that $\nabla \times\left(Q_{k+1}^{[k-1, k]} \times Q_{k+1}^{[k, k-1]} \times\{0\}\right) \subset \overline{\mathcal{U}}_{k}^{(2)}$. Next, we apply (A.6) to $\left(\{0\} \times\{0\} \times Q_{k+2}^{[k-1, k-1]}\right)$ to see that this is also a subset of $\overline{\mathcal{U}}_{k}^{(2)}$. These two set inclusions show that $\mathcal{R}_{k}^{(2)} \subset \overline{\mathcal{U}}_{k}^{(2)}$.

Another application of (A.6) gives $\mathcal{R}_{k}^{(3)}=Q_{k+3}^{[k-1, k-1]} \subseteq Q_{k+3}^{k-1, k-1, k-1}=\overline{\mathcal{U}}_{k}^{(3)}$.

Finally, Lemma 4.5 shows that each $\mathcal{R}_{k}^{(s)}$ also satsifies the trace constraints of of the $\mathcal{U}_{k}^{(s)}$.

As suggested in the preamble to Theorem 4.7, we can reuse the interpolation operators from the old spaces, (A.4), to create interpolation operators for the new spaces. Since the trace spaces of $\mathcal{R}_{k}^{(s)}$ are the same as $\mathcal{U}_{k}^{(s)}$, we just need to define projections $\Xi_{k, K}^{(s)}: \mathcal{U}_{k}^{(s)}(K) \rightarrow \mathcal{R}_{k}^{(s)}(K)$ that do not change the trace data. We denote the subspace of all shape-functions in $\mathcal{R}_{k}^{(s)}(K)$ with zero trace as $\mathcal{R}_{k, 0}^{(s)}(K)$.

Definition A.1. For $u \in \mathcal{U}_{k}^{(s)}(K)$, define $\Xi_{k, K}^{(s)}: \mathcal{U}_{k}^{(s)}(K) \rightarrow \mathcal{R}_{k}^{(s)}(K)$ as

$$
\Xi_{k, K}^{(s)} u:=v_{u}+w_{u}
$$


where $v_{u} \in \mathcal{R}_{k}^{(s)}(K)$ is some function satisfying $\left.v_{u}\right|_{\partial K}=\left.u\right|_{\partial K}$ and $w_{u} \in \mathcal{R}_{k, 0}^{(s)}(K)$ is the minimizer of the functional $v \rightarrow\left\|d\left(u-v_{u}-v\right)\right\|_{0}$ over the admissible set $\mathcal{A}_{k, K}^{(s)}$, defined as:

$$
\begin{aligned}
& \mathcal{A}_{k, K}^{(0)}:=\mathcal{R}_{k, 0}^{(0)}(K) \\
& \mathcal{A}_{k, K}^{(s)}:=\left\{v \in \mathcal{R}_{k, 0}^{(s)}(K):(v, d w)=0 \forall w \in \mathcal{R}_{k, 0}^{(s-1)}(K)\right\}, s=1,2,3 .
\end{aligned}
$$

Lemma 4.5 means that the trace spaces of $\mathcal{R}_{k}^{(s)}(K)$ and $\mathcal{U}_{k}^{(s)}(K)$ are identical, so it is always possible to find an extension, $v_{u}$. The spaces $\mathcal{A}_{k, K}^{(s)}$ are non-empty because they always contain the zero-element so there always exists a minimiser, $w_{u}$. The uniqueness of $w_{u}$ (for a given choice of $v_{u}$ ) can be established using a Friedrichs-type inequality and it is then clear that $\Xi_{k, K}^{(s)} u$ is independent of the choice of $v_{u}$.

In fact, the operators $\Xi_{k, K}^{(s)} u$ are just the projection-based interpolants of $\mathcal{U}_{k}^{(s)}(K)$ onto $\mathcal{R}_{k}^{(s)}(K)$. More details of projection-based interpolation can be found in [8], which also establishes the important commutativity property: $\Xi_{k, K}^{(s+1)} \circ d=d \circ \Xi_{k, K}^{(s)}$.

The proof of Theorem 4.7 now follows easily:

Proof. Define the maps $\Phi_{k, K}^{(s)}: H^{2} \Lambda(s)(K) \rightarrow \mathcal{R}_{k}^{(s)}(K)$ as

$$
\Phi_{k, K}^{(s)}=\Xi_{k, K}^{(s)} \circ \Pi_{k, K}^{(s)} .
$$

Since both $\Xi_{k, K}^{(s)}$ and $\Pi_{k, K}^{(s)}$ commute with $d$, so does $\Phi_{k, K}^{(s)}$.

Of course, defining an interpolation operator is equivalent to defining degrees of freedom. Whereas the old elements used bases for Helmholtz decompositions of $\mathcal{U}_{k, 0}^{(s)}(K)$ as test functions for the degrees; the new elements require Helmholtz decompositions of $\mathcal{R}_{k, 0}^{(s)}(K)$; these can readily be determined from the full-space Helmholtz decomposition implied in the definitions, (4.3). The external degrees of freedom for both sets of elements are identical. Note also that if $\Pi_{k, K}^{(s)}$ were a projection based interpolant, then $\Phi_{k, K}^{(s)}$ would be too.

Finally, we prove Theorem 4.10:

Proof. Each $\mathcal{X}_{r, k}^{(s)}$ is a subset of $\mathcal{R}_{k}^{(s)}$, so

$$
\mathcal{X}_{0, k}^{(s)}(K) \oplus \cdots \oplus \mathcal{X}_{k, k}^{(s)}(K) \subset \mathcal{R}_{k}^{(s)}(K) .
$$

For the reverse inclusion, we will deal with each $s \in\{0,1,2,3\}$, in turn. For every $s \in\{0,1,2,3\}$, the transformation rule, (3.5), gives $\hat{u} \circ \phi=w_{\phi}^{(s)} \tilde{u}$.

For 0 -forms, the weight in the change of coordinates formula $w_{\phi}^{(0)}$ is equal to 1 so any $u \in \mathcal{R}_{k}^{(s)}(K)$ satisfies $\hat{u} \circ \phi=\tilde{u} \in \mathcal{R}_{k}^{(0)}=Q_{k}^{[k, k]}$. The decomposition, (4.2) gives

$$
Q_{k}^{[k, k]}=Q_{0}^{0,0,0} \oplus \cdots \oplus Q_{k}^{k, k, 0}
$$

which is a subset of $Q_{0}^{1,1,0} \oplus \cdots \oplus Q_{k}^{k+1, k+1,0}$ so $u \in \mathcal{X}_{0, k}^{(0)}(K) \oplus \cdots \oplus \mathcal{X}_{k, k}^{(s)}(K)$.

When $s=1$ we will consider a basis for $\mathcal{R}_{k}^{(1)}(K)$ and show that each element, $u$, of the basis is a member of $\mathcal{X}_{r, k}^{(1)}(K)$ for some $r \in\{0 \ldots k\}$, which amounts to showing that each $u_{\hat{\imath}} \circ \phi \in Q_{r}^{r+1, r+1,0}$.

From the definition given in (4.3c) it is natural to consider three cases for an element of a basis for $\mathcal{R}_{k}^{(1)}(K)$. First suppose that $\tilde{u} \in\left(Q_{k+1}^{[k-1, k]} \times 0 \times 0\right)$ with $u_{\tilde{1}}=\frac{x^{a} y^{b}}{(1+z)^{c}}$. From the definition of $Q_{k+1}^{[k-1, k]}$ we see that 
$0 \leq a \leq c-2$ and $0 \leq b \leq c-1$ and so $2 \leq c \leq k+1$. Then $w_{\phi}^{(1)} \tilde{u}=\left(\frac{x^{a} y^{b}}{(1+z)^{c-1}}, 0, \frac{x^{a+1} y^{b}}{(1+z)^{c-1}}\right)^{t}$ and so each $u_{\hat{\imath}} \in Q_{c-1}^{a+1, b, 0} \subset Q_{r}^{r, r, 0}$ where $r=c-1 \in\{1 \ldots k\}$. The second case is when $\tilde{u} \in\left(0 \times Q_{k+1}^{[k, k-1]} \times 0\right)$ and the reasoning is identical to the first. Finally suppose that $\tilde{u}=\nabla p$ where $p=\frac{x^{a} y^{b}}{(1+z)^{c}} \in Q_{k}^{[k, k]}$. If $c=0, p=1$ and therefore $\nabla p=0$. So we can take $c \geq 1$ and see that each entry of

$$
w_{\phi}^{(1)} \tilde{u}=\left(\begin{array}{c}
a \frac{x^{a-1} y^{b}}{(1+z)^{c-1}} \\
b \frac{x^{a} b^{-1}}{(1+z)^{c-1}} \\
(a+b-c) \frac{x^{a} y^{b}}{(1+z)^{c-1}}
\end{array}\right)
$$

is in $Q_{r}^{r+1, r+1,0}$ for some $r \in\{0 \ldots k\}$.

When $u \in \mathcal{R}_{k}^{(2)}(K)$, let us start with the case $\tilde{u} \in\left(0 \times 0 \times Q_{k+2}^{[k-1, k-1]}\right)$ and write $u_{\tilde{3}}=\frac{x^{a} y^{b}}{(1+z)^{c}}$. Again, it is simple to check that each of the entries in the vector $w_{\phi}^{(2)} \tilde{u}=\left(-\frac{x^{a+1} y^{b}}{(1+z)^{c-2}},-\frac{x^{a} y^{b+1}}{(1+z)^{c-2}}, \frac{x^{a} y^{b}}{(1+z)^{c-2}}\right)^{t}$ is in $Q_{r}^{r+1, r+1,0}$ for some $r \in\{0 \ldots k\}$. Now suppose that $\tilde{u}=\nabla \times \tilde{v}$ where $\tilde{v} \in\left(Q_{k+1}^{[k-1, k]} \times 0 \times 0\right)$ with $v_{\tilde{1}}=\frac{x^{a} y^{b}}{(1+z)^{c}}$. From the $s=1$ case, we know that $c \geq 2$ and so it is straightforward to verify that each of the entries in

$$
w_{\phi}^{(2)} \tilde{u}=(1+z)^{2}\left(\begin{array}{ccc}
1+z & 0 & -x \\
0 & 1+z & -y \\
0 & 0 & 1
\end{array}\right)\left(\begin{array}{c}
0 \\
\frac{-c x^{a} y^{b}}{(1+z)^{c+1}} \\
\frac{b x^{a} y^{b-1}}{(1+z)^{c}}
\end{array}\right)=\left(\begin{array}{c}
\frac{-b x^{a+1} y^{b}}{(1+z)^{c-2}} \\
\frac{-c x^{a} y^{b}}{(1+z)^{c-2}}+\frac{-b x^{a} y^{b}}{(1+z)^{c-2}} \\
\frac{b x^{a} y^{b-1}}{(1+z)^{c-2}}
\end{array}\right)
$$

are in $Q_{r}^{r+1, r+1,0}$ for some $r \in\{0 \ldots k\}$. The argument for $\tilde{u}=\nabla \times \tilde{v}$ with $\tilde{v} \in\left(0 \times Q_{k+1}^{[k, k-1]} \times 0\right)$ is the same.

Finally, $u \in \mathcal{R}_{k}^{(3)}(K)$ means that $\tilde{u} \in Q_{k+3}^{[k-1, k-1]}$. The weight $w_{\phi}^{(3)}=\frac{1}{(1+z)^{4}}$ so $\hat{u} \circ \phi=\frac{1}{(1+z)^{4}} \tilde{u} \in Q_{k-1}^{[k-1, k-1]}$ and the reasoning is the same as the 0 -form case.

\section{REFERENCES}

[1] D.N. Arnold, R.S. Falk and R. Winther, Finite element exterior calculus, homological techniques, and applications. Acta Num. 15 (2006) 1-155.

[2] D.N. Arnold, R.S. Falk and R. Winther, Finite element exterior calculus: from Hodge theory to numerical stability. Bull. Am. Math. Soc. 47 (2010) 281-354.

[3] M. Bergot, G. Cohen and M. Duruflé, Higher-order finite elements for hybrid meshes using new nodal pyramidal elements. J. Sci. Comput. 42 (2010) 345-381.

[4] J.H. Bramble and S.R. Hilbert, Estimation of linear functionals on Sobolev spaces with application to Fourier transforms and spline interpolation. SIAM J. Numer. Anal. 7 (1970) 112-124.

[5] S.C. Brenner and L.R. Scott, The mathematical theory of finite element methods. Springer Verlag (2008).

[6] P.G. Ciarlet, The Finite Element Method for Elliptic Problems. Society for Industrial Mathematics (2002).

[7] J.L. Coulomb, F.X. Zgainski and Y. Maréchal, A pyramidal element to link hexahedral, prismatic and tetrahedral edge finite elements. IEEE Trans. Magn. 33 (1997) 1362-1365.

[8] L. Demkowicz and A. Buffa, $H^{1}, H$ (curl) and $H$ (div)-conforming projection-based interpolation in three dimensions. Quasioptimal p-interpolation estimates. Comput. Methods Appl. Mech. Eng. 194 (2005) 267-296.

[9] L. Demkowicz, J. Kurtz, D. Pardo, M. Paszenski and W. Rachowicz, Computing with hp-Adaptive Finite Elements Frontiers: Three Dimensional Elliptic and Maxwell Problems with Applications 2. Chapman \& Hall (2007).

[10] M. Fortin and F. Brezzi, Mixed and Hybrid Finite Element Methods (Springer Series in Computational Mathematics). SpringerVerlag Berlin and Heidelberg GmbH \& Co. K (1991). 
[11] V. Gradinaru and R. Hiptmair, Whitney elements on pyramids. Electronic Transactions on Numerical Analysis 8 (1999) $154-168$.

[12] R.D. Graglia and I.L. Gheorma, Higher order interpolatory vector bases on pyramidal elements. IEEE Trans. Antennas Propag. 47 (1999) 775.

[13] P.C. Hammer, O.J. Marlowe and A.H. Stroud, Numerical integration over simplexes and cones. Mathematical Tables Aids Comput. 10 (1956) 130-137.

[14] J.M. Melenk, K. Gerdes and C. Schwab, Fully discrete hp-finite elements: Fast quadrature. Comput. Methods Appl. Mech. Eng. 190 (2001) 4339-4364.

[15] P. Monk, Finite element methods for Maxwell's equations. Numerical Mathematics and Scientific Computation. Oxford University Press, New York (2003).

[16] J.-C. Nedéléc, Mixed finite elements in $\mathbb{R}^{3}$. Num. Math. 35 (1980) 315-341.

[17] N. Nigam and J. Phillips, High-order conforming finite elements on pyramids. IMA J. Numer. Anal. (2011); doi: 10.1093/imanum/drr015.

[18] A.H. Stroud, Approximate calculation of multiple integrals. Prentice-Hall Inc., Englewood Cliffs, N.J. (1971).

[19] J. Warren, On the uniqueness of barycentric coordinates, in Topics in Algebraic Geometry and Geometric Modeling: Workshop on Algebraic Geometry and Geometric Modeling, July 29-August 2, 2002, Vilnius University, Lithuania. American Mathematical Society 334 (2002) 93-99.

[20] C. Wieners, Conforming discretizations on tetrahedrons, pyramids, prisms and hexahedrons. Technical report, University of Stuttgart.

[21] S. Zaglmayr, High Order Finite Element methods for Electromagnetic Field Computation. Ph. D. thesis, Johannes Kepler University, Linz (2006).

[22] F.-X. Zgainski, J.-L. Coulomb, Y. Marechal, F. Claeyssen and X. Brunotte, A new family of finite elements: the pyramidal elements. IEEE Trans. Magn. 32 (1996) 1393-1396. 